MATHEMATICS OF COMPUTATION

Volume 71, Number 239, Pages 1105-1135

S $0025-5718(01) 01344-8$

Article electronically published on November 14, 2001

\title{
CONVERGENCE RATE ANALYSIS OF AN ASYNCHRONOUS SPACE DECOMPOSITION METHOD FOR CONVEX MINIMIZATION
}

\author{
XUE-CHENG TAI AND PAUL TSENG
}

\begin{abstract}
We analyze the convergence rate of an asynchronous space decomposition method for constrained convex minimization in a reflexive Banach space. This method includes as special cases parallel domain decomposition methods and multigrid methods for solving elliptic partial differential equations. In particular, the method generalizes the additive Schwarz domain decomposition methods to allow for asynchronous updates. It also generalizes the BPX multigrid method to allow for use as solvers instead of as preconditioners, possibly with asynchronous updates, and is applicable to nonlinear problems. Applications to an overlapping domain decomposition for obstacle problems are also studied. The method of this work is also closely related to relaxation methods for nonlinear network flow. Accordingly, we specialize our convergence rate results to the above methods. The asynchronous method is implementable in a multiprocessor system, allowing for communication and computation delays among the processors.
\end{abstract}

\section{INTRODUCTION}

With the advent of multiprocessor computing systems, there has been much work in the design and analysis of iterative methods that can take advantage of the parallelism to solve large linear and nonlinear algebraic problems. In these methods, the computation per iteration is distributed over the processors and each processor communicates the result of its computation to the other processors. In some systems, the activities of the processors are highly synchronized (possibly via a central processor), while in other systems (typically those with many processors), the processors may experience communication or computation delays. The latter lack of synchronization makes the analysis of the methods much more difficult. To aid in this analysis, Chazan and Miranker [16] proposed a model of asynchronous computation that allows for communication and computation delays among processors, and they showed that the Jacobi method for solving a diagonally dominant

Received by the editor September 27, 2000.

2000 Mathematics Subject Classification. Primary 65J10, 65M55, 65Y05; Secondary 65K10, $65 \mathrm{~N} 55$.

Key words and phrases. Convex minimization, space decomposition, asynchronous computation, convergence rate, domain decomposition, multigrid, obstacle problem.

The work of the first author was supported by the Norwegian Research Council Strategic Institute Program within Inverse Problems at RF-Rogaland Research, and by Project SEP-115837/431 at Mathematics Institute, University of Bergen.

The work of the second author was supported by the National Science Foundation, Grant No. CCR-9311621. 
system of linear equations converges under this model of asynchronous computation. Subsequently, there has been extensive study of asynchronous methods based on such a model (see [5, 6] and references therein). For these methods, convergence typically requires the algorithmic mapping to be either isotone or nonexpansive with respect to the $L^{\infty}$-norm or gradient-like. However, aside from the easy case where the algorithmic mapping is a contraction with respect to the $L^{\infty}$-norm, there have been few studies of the convergence rate of these methods. One such study was done in [55] for an asynchronous gradient-projection method.

In this paper, we study the convergence rate of asynchronous Jacobi and GaussSeidel type methods for finite- or infinite-dimensional convex minimization of the form

$$
\min _{v_{i} \in K_{i}, i=1, \ldots, m} F\left(\sum_{i=1}^{m} v_{i}\right),
$$

where each $K_{i}$ is a nonempty closed convex set in a real reflexive Banach space $V$ and $F$ is a real-valued lower semicontinuous Gâteau-differentiable function that is strongly convex on $\sum_{i=1}^{m} K_{i}$. Our interest in these methods stems from their close connection to relaxation methods for nonlinear network flow (see [4, 5, 56] and references therein) and to domain decomposition (DD) and multigrid (MG) methods for solving elliptic partial differential equations (see [7, 8, 9, 14, 18, 19. 33 40, 45, 52, 53, 57 and references therein). For example, the additive and the multiplicative Schwarz methods may be viewed as Jacobi and Gauss-Seidel type methods applied to linear elliptic partial differential equations reformulated as (1) 9, 57. DD and MG methods are also useful as preconditioners and it can be shown that such preconditioning improves the condition number of the discrete approximation [7, 8, 10, 9, 14, 33, 40, 45, 57. In addition, DD and MG methods are well suited for parallel implementation, for which both synchronous and asynchronous versions have been proposed. Of the work on asynchronous methods 21, 22, 27, 38, 37, 39, 46, we especially mention the numerical tests by Frommer et al. 22] which showed that, through improved load balancing, asynchronous methods can be advantageous in solving even simple linear equations. Although these tests did not use the coarse mesh in its implementation of the DD method, it is plausible that the asynchronous method would still be advantageous when the coarse mesh is used. However, the convergence rate analysis of the above asynchronous methods seems still missing from the literature. In the case where the equation is linear (corresponding to $F$ being quadratic and $K_{1}, \ldots, K_{m}$ being suitable subspaces of $V$ ) or almost linear, this issue has been much studied for synchronous methods (see see [7, 8, 9, 14, 18, 19, 33, 40, 45, 52, 53, 57] and references therein) but little studied for asynchronous methods. In the case where the equation is generally nonlinear (corresponding to $K_{1}, \ldots, K_{m}$ being suitable subspaces of $V$ ), there are some convergence studies for synchronous methods [15, [18, 44, [52, [53], and none for asynchronous methods. In the case where $K_{1}, \ldots, K_{m}$ are not all subspaces, there are various convergence studies for synchronous methods (see [1, $12,23,25,28,29,30,31,34,35,36,47,50$, and references therein) but, again, none for asynchronous methods.

The contributions of the present work are two-fold.

- We consider an asynchronous version of Jacobi and Gauss-Seidel methods for solving (1), and we show that, under a Lipschitzian assumption on the 
Gâteau derivative $F^{\prime}$ and a norm equivalence assumption on the product of $K_{1}, \ldots, K_{m}$ and their sum (see (5) and (6i)), this asynchronous method attains a global linear rate of convergence with a convergence factor that can be explicitly estimated (see Theorem (1). This provides a unified convergence and convergence rate analysis for such asynchronous methods.

- We apply the above convergence result to (finite-dimensional) linearly constrained convex programs and, in particular, nonlinear network flow problems. This yields convergence rate results for some asynchronous network relaxation methods (see Section 6). Previous work studied the convergence of these methods, but no rate of convergence result was obtained. We also apply the above convergence result to certain nonlinear elliptic partial differential equations. This yields convergence rate results for some asynchronous parallel DD and MG methods for solving these equations and, in particular, the convergence factor is shown not to depend on the mesh parameters (see Section 7). When implementing multigrid methods on parallel processors, the nodal basis is often organized into different groups. The computation within each group can be sequential while the computation in different groups could be done in parallel. The asynchronous convergence rate analysis provides a convergence rate estimate when computation in different groups is not fully synchronized. Lastly, application to an overlapping DD method for obstacle problems is studied. We show that the method attains a linear rate of convergence with a convergence factor depending on the overlapping size, but not on the mesh size or the number of subdomains.

We note that alternative approaches such as Newton-type methods have also been applied to develop synchronous DD and MG methods for nonlinear partial differential equations without constraints [2, 3, 11, 26, 41, 58, 59. However, these methods use the traditional DD and MG approach or use a special two-grid treatment. Our approach is different even for nonlinear partial differential equations without constraints.

\section{Problem Description AND SPACE DECOMPOSITION}

Let $V$ be a real reflexive Banach space with norm $\|\cdot\|$ and let $V^{\prime}$ be its dual space, i.e., the space of all real-valued linear continuous functionals on $V$. The value of $f \in V^{\prime}$ at $v \in V$ will be denoted by $\langle f, v\rangle$, i.e., $\langle\cdot, \cdot\rangle$ is the duality pairing of $V$ and $V^{\prime}$. We wish to solve the minimization problem

$$
\min _{v \in K} F(v),
$$

where $K$ is a nonempty closed (in the strong topology) convex set in $V$ and $F: V \mapsto \Re$ is a lower semicontinuous convex Gâteau-differentiable function. We assume $F$ is strongly convex on $K$ or, equivalently, its Gâteau derivative $\lim _{t \rightarrow 0}(F(v+t w)-F(v)) / t$, which is a well-defined linear continuous functional of $w$ denoted by $F^{\prime}(v)$ (so $F^{\prime}: V \mapsto V^{\prime}$ ), is strongly monotone on $K$, i.e.,

$$
\left\langle F^{\prime}(u)-F^{\prime}(v), u-v\right\rangle \geq \sigma\|u-v\|^{2}, \quad \forall u, v \in K,
$$

where $\sigma>0$. It is known that, under the above assumptions, (2) has a unique solution $\bar{u}$ [24, p. 23]. 
We assume that the constraint set $K$ can be decomposed as the Minkowski sum

$$
K=\sum_{i=1}^{m} K_{i}
$$

for some nonempty closed convex sets $K_{i}$ in $V, i=1, \ldots, m$. This means that, for any $v \in K$, we can find $v_{i} \in K_{i}$, not necessarily unique, satisfying $\sum_{i=1}^{m} v_{i}=v$ and, conversely, for any $v_{i} \in K_{i}, i=1 \ldots, m$, we have $\sum_{i=1}^{m} v_{i} \in K$. Following Xu [57], we call (4) a space decomposition of $K$, with the term "space" used loosely here. Then we may reformulate (2) as the minimization problem (11), with $\left(\bar{u}_{1}, \ldots, \bar{u}_{m}\right)$ being a solution (not necessarily unique) of (1) if and only if $\bar{u}_{i} \in K_{i}$ for $i=1, \ldots, m$ and $\sum_{i=1}^{m} \bar{u}_{i}=\bar{u}$. As was noted earlier, the reformulated problem (1) is of interest because methods such as DD and MG methods may be viewed as Jacobi and GaussSeidel methods for its solution. The method we study will be an asynchronous version of these methods. The above reformulation was proposed in [9, 57] (for the case where $F$ is quadratic and $K=V$ ) to give a unified analysis of DD and MG methods for linear elliptic partial differential equations. The general case was treated in 47, 50] (also see [48, 52, for the case of $K=V$ ).

For the above space decomposition, we will assume that there is a constant $C_{1}>0$ such that for any $v_{i} \in K_{i}, i=1, \ldots, m$, there exists $\bar{u}_{i} \in K_{i}$ satisfying

$$
\bar{u}=\sum_{i=1}^{m} \bar{u}_{i} \quad \text { and } \quad\left(\sum_{i=1}^{m}\left\|\bar{u}_{i}-v_{i}\right\|^{2}\right)^{\frac{1}{2}} \leq C_{1}\left\|\bar{u}-\sum_{i=1}^{m} v_{i}\right\|
$$

(see [14, p. 95], [50, 52], [57, Lemma 7.1] for similar assumptions). We will also assume $F^{\prime}$ has a weak Lipschitzian property in the sense that there is a constant $C_{2}>0$ such that

(6)

$$
\begin{gathered}
\sum_{i=1}^{m} \sum_{j=1}^{m}\left\langle F^{\prime}\left(w_{i j}+u_{i j}\right)-F^{\prime}\left(w_{i j}\right), v_{i}\right\rangle \leq C_{2}\left(\sum_{j=1}^{m} \max _{i=1, \ldots, m}\left\|u_{i j}\right\|^{2}\right)^{\frac{1}{2}}\left(\sum_{i=1}^{m}\left\|v_{i}\right\|^{2}\right)^{\frac{1}{2}} \\
\forall w_{i j} \in K, u_{i j} \in K_{j}^{\ominus}, v_{i} \in K_{i}^{\ominus}, i, j=1, \ldots, m
\end{gathered}
$$

where we define the set difference $K_{i}^{\ominus}=\left\{u-v: u, v \in K_{i}\right\} \subset V$. The above assumption generalizes those in 50,52, 53 for the case of $K_{i}$ being a subspace, for which $K_{i}^{\ominus}=K_{i}$.

Furthermore, we will paint each of the sets $K_{1}, \ldots, K_{m}$ one of $c$ colors, with the colors numbered from 1 up to $c$, such that sets painted the same color $k \in\{1, \ldots, c\}$ are orthogonal in the sense that

$$
\begin{aligned}
\left\|\sum_{i \in I(k)} v_{i}\right\|^{2}= & \sum_{i \in I(k)}\left\|v_{i}\right\|^{2}, \forall v_{i} \in K_{i}^{\ominus}, i \in I(k), \\
\left\langle F^{\prime}\left(u+\sum_{i \in I(k)} v_{i}\right), \sum_{i \in I(k)} v_{i}\right\rangle \leq & \sum_{i \in I(k)}\left\langle F^{\prime}\left(u+v_{i}\right), v_{i}\right\rangle, \\
& \forall u \in K, v_{i} \in K_{i}^{\ominus}, i \in I(k),
\end{aligned}
$$

where $I(k)=\left\{i \in\{1, \ldots, m\}: K_{i}\right.$ is painted color $\left.k\right\}$ (see [14, §4.1], [53] for similar orthogonal decompositions in the case $K_{i}$ is a subspace). Thus $I(1), \ldots, I(c)$ are disjoint subsets of $\{1, \ldots, m\}$ whose union is $\{1, \ldots, m\}$ and $I(k)$ comprises the indexes of the sets painted the color $k$. Although $c=m$ is always a valid choice, in 
some of the applications that we will consider, it is essential that $c$ be independent of $m$. In the context of a network flow problem, each set $K_{i}$ may correspond to a node of the network and sets are painted different colors if their corresponding nodes are joined by an arc. In the context of a partial differential equation defined on a domain $\Omega \subset \Re^{d}$, each set $K_{i}$ may correspond to a subdomain of $\Omega$ and sets are painted different colors if their corresponding subdomains intersect (see Sections 6 and 7 for details).

Remark 1. It can be seen that condition ([6) is implied by the following strengthened Cauchy-Schwarz inequality (also see [45, p. 155], [57] for the case of quadratic $F$ and subspace $K_{i}$ ):

$$
\left\langle F^{\prime}\left(w_{i j}+u_{i j}\right)-F^{\prime}\left(w_{i j}\right), v_{i}\right\rangle \leq \epsilon_{i j}\left\|u_{i j}\right\|\left\|v_{i}\right\|, \quad \forall w_{i j} \in K, u_{i j} \in K_{j}^{\ominus}, v_{i} \in K_{i}^{\ominus},
$$

with $C_{2}$ being the spectral radius of the matrix $\mathcal{E}=\left[\epsilon_{i j}\right]_{i, j=1}^{m}$, assumed to be symmetric.

Remark 2. For locally strongly convex problems, the constants $\sigma, C_{1}, C_{2}$ may depend on $u, v, v_{i}, w_{i j}, u_{i j}$. In this case, the subsequent analysis should be viewed as being local in nature, i.e., it is valid when the iterated solutions lie in a neighborhood of the true solution (see Section 7).

\section{An ASYNCHRONOUS SPACE DECOMPOSITION METHOD}

Since $F$ is lower semicontinuous and strongly convex, for each $\left(u_{1}, \ldots, u_{m}\right) \in$ $K_{1} \times \cdots \times K_{m}$ and each $i \in\{1, \ldots, m\}$, there exists a unique $w_{i} \in K_{i}$ satisfying

$$
F\left(\sum_{j \neq i} u_{j}+w_{i}\right) \leq F\left(\sum_{j \neq i} u_{j}+v_{i}\right), \quad \forall v_{i} \in K_{i}
$$

(see [24, p. 23]). Let $\pi_{i}\left(u_{1}, \ldots, u_{m}\right)$ denote this $w_{i}$. Then $\left(\pi_{1}, \ldots, \pi_{m}\right)$ may be viewed as the algorithmic mapping associated with the block Jacobi method for solving (1). Consider an asynchronous version of the block Jacobi method, parameterized by a stepsize $\gamma \in(0,1]$, which for simplicity we assume to be fixed, that generates a sequence of iterates $\left(u_{1}(t), \ldots, u_{m}(t)\right), t=0,1, \ldots$, with $\left(u_{1}(0), \ldots, u_{m}(0)\right) \in K_{1} \times \cdots \times K_{m}$ given, according to the updating formula,

$$
u_{i}(t+1)=u_{i}(t)+\gamma s_{i}(t), \quad i=1, \ldots, m,
$$

where we define

$$
\begin{aligned}
s_{i}(t) & = \begin{cases}w_{i}(t)-u_{i}(t) & \text { if } t \in T^{i} \\
0 & \text { otherwise }\end{cases} \\
w_{i}(t) & =\pi_{i}\left(u_{1}\left(\tau_{1}^{i}(t)\right), \ldots, u_{m}\left(\tau_{m}^{i}(t)\right)\right)
\end{aligned}
$$

and $T^{i}$ is some subset of $\{0,1, \ldots\}$ and each $\tau_{j}^{i}(t)$ is some nonnegative integer not exceeding $t$. Since each $K_{i}$ is convex and $\gamma \in(0,1]$, an induction argument shows that $\left(u_{1}(t), \ldots, u_{m}(t)\right) \in K_{1} \times \cdots \times K_{m}$ for all $t=0,1, \ldots$.

We will assume that the iterates are updated in a partially asynchronous manner [5, Chap. 7], i.e., there exists an integer $B \geq 1$ such that

$$
\begin{array}{ll}
\{t, t+1, \ldots, t+B-1\} \cap T^{i} \neq \emptyset, & t=0,1, \ldots, \forall i, \\
0 \leq t-\tau_{j}^{i}(t) \leq B-1 \quad \text { and } \tau_{i}^{i}(t)=t, & \forall t \in T^{i}, \forall i, j .
\end{array}
$$


We say that a color $k \in\{1, \ldots, c\}$ is active at time $t$ if there exists an $i \in I(k)$ such that $t \in T^{i}$. Recall that $I(k)$ indexes those sets painted the color $k$. Denoting by $c_{t}$ the total number of colors that are active at time $t$, we will also assume that

$$
\gamma<\min \left\{\frac{\sigma}{2 C_{2} B}, \frac{1}{c_{t}}\right\}, \quad t=0,1, \ldots
$$

Notice that $\gamma$ does not depend on $m$ nor on $C_{1}$. Although (15) may give a very conservative value of $\gamma$, this can be remedied by starting with a larger $\gamma$ and decreasing $\gamma$ whenever "sufficient progress" (defined in any reasonable way) is not made and (15) is not satisfied.

Remark 3. The above asynchronous method models a situation in which computation is distributed over $m$ processors with the $i$ th processor being responsible for updating $u_{i}$ and communicating the updated value to the other processors. $T^{i}$ is the set of "times" at which $u_{i}$ is updated by processor $i$ (by applying $\pi_{i}$ to its current copy of $\left.\left(u_{1}, \ldots, u_{m}\right)\right) ; u_{i}(t)$ is the value of $u_{i}$ known to processor $i$ at time $t$; and $\tau_{j}^{i}(t)$ is the time at which the value of $u_{j}$ used by processor $i$ at time $t$ is generated by processor $j$, so $t-\tau_{j}^{i}(t)$ is the communication delay from processor $j$ to processor $i$ at time $t$. Thus, the processors need not wait for each other when updating $\left(u_{i}\right)_{i=1}^{m}$, and the values used in the computation may be out-of-date.

Remark 4 . The assumption that $\tau_{i}^{i}(t)=t$ can perhaps be removed through a more careful analysis, though this seems to be a reasonable assumption in practice. Intuitively, (13) says that each component $u_{i}$ is updated at least once every $B$ time units, and (14) says that the information used by processor $i$ from processor $j$ should not be out-of-date by more than $B$ time units. This assumption of bounded communication and computation delay is needed for a convergence rate analysis.

\section{Convergence rate of the asynchronous method}

In this section we prove that the iterates $\left(u_{1}(t), \ldots, u_{m}(t)\right), t=0,1, \ldots$, generated by the asynchronous method (10)-(15) attain linear rate of convergence, with a factor that depends on $\sigma, C_{1}, C_{2}, c$ and $B, \gamma$ only (see Theorem 11). While parts of our proof use ideas from the analysis of asynchronous gradient-like methods 5 . $\S 7.5]$, [55, a number of new proof ideas are introduced to account for different problem assumptions and different natures of the Jacobi and Gauss-Seidel algorithmic mappings. To simplify the notation in our analysis, define

$$
u(t)=\sum_{j=1}^{m} u_{j}(t), \quad z_{i}(t)=\sum_{j=1}^{m} u_{j}\left(\tau_{j}^{i}(t)\right),
$$

for all $i$ and $t$. If $t \in T^{i}$, then the definition (12) of $w_{i}(t)$ and the fact that $\tau_{i}^{i}(t)=t$ and $F$ is Gâteau-differentiable imply $w_{i}(t)$ satisfy the optimality condition

$$
\left\langle F^{\prime}\left(z_{i}(t)+w_{i}(t)-u_{i}(t)\right), v_{i}-w_{i}(t)\right\rangle \geq 0, \quad \forall v_{i} \in K_{i} .
$$

Our analysis will be based on estimates given in the following two key lemmas.

Lemma 1 (Descent estimate). Let $A_{1}$ and $A_{2}$ be defined by

$$
A_{2}=\frac{C_{2}^{2} B^{2}}{\sigma}, \quad A_{1}=\frac{\sigma}{4}-\gamma^{2} A_{2} .
$$


For $t=0,1, \ldots$, we have

$$
F(u(t+B)) \leq F(u(t))-\gamma A_{1} \sum_{j=1}^{m} \sum_{\tau=t}^{t+B-1}\left\|s_{j}(\tau)\right\|^{2}+\gamma^{3} A_{2} \sum_{j=1}^{m} \sum_{\tau=t-B+1}^{t-1}\left\|s_{j}(\tau)\right\|^{2} .
$$

Proof. Fix any time $t \in\{0,1, \ldots\}$. Recall that $c_{t}$ is the total number of colors active at time $t$ and, without loss of generality, we assume that the first $c_{t}$ colors are active. Then $s_{i}(t)=0$ for all $i \in I(k)$ and $k>c_{t}$, so by defining

$$
e_{k}(t)=\sum_{i \in I(k)} s_{i}(t)
$$

and using (16), (10) and the convexity of $F$, we have

$$
\begin{aligned}
F(u(t+1)) & =F\left(u(t)+\gamma \sum_{i=1}^{m} s_{i}(t)\right) \\
& =F\left(u(t)+\gamma \sum_{k=1}^{c_{t}} \sum_{i \in I(k)} s_{i}(t)\right) \\
& =F\left(\left(1-c_{t} \gamma\right) u(t)+\sum_{k=1}^{c_{t}} \gamma\left(u(t)+e_{k}(t)\right)\right) \\
& \leq\left(1-c_{t} \gamma\right) F(u(t))+\gamma \sum_{k=1}^{c_{t}} F\left(u(t)+e_{k}(t)\right) \\
& =F(u(t))+\gamma \sum_{k=1}^{c_{t}}\left(F\left(u(t)+e_{k}(t)\right)-F(u(t))\right) .
\end{aligned}
$$

Since $u(t) \in K$ and $u(t)+e_{k}(t) \in K$, the strong monotonicity of $F^{\prime}$ on $K$ given in (3) implies

$$
F(u(t)) \geq F\left(u(t)+e_{k}(t)\right)-\left\langle F^{\prime}\left(u(t)+e_{k}(t)\right), e_{k}(t)\right\rangle+\frac{\sigma}{2}\left\|e_{k}(t)\right\|^{2} .
$$

Define

$$
\phi_{j}^{i}(t)=\sum_{k=1}^{j} u_{k}\left(\tau_{k}^{i}(t)\right)+\sum_{k=j+1}^{m} u_{k}(t), \quad j=0,1, \ldots, m
$$

Then $\phi_{0}^{i}(t)=u(t)$ and $\phi_{m}^{i}(t)=z_{i}(t)$ and

$$
\phi_{j}^{i}(t)-\phi_{j-1}^{i}(t)=u_{j}\left(\tau_{j}^{i}(t)\right)-u_{j}(t) \in K_{j}^{\ominus}, \quad j=1, \ldots, m .
$$

If $t \in T^{i}$, then setting $v_{i}=u_{i}(t)$ in (17) and noting that $s_{i}(t)=w_{i}(t)-u_{i}(t)$ (see (11), we obtain that

$$
\begin{aligned}
0 \leq & -\left\langle F^{\prime}\left(z_{i}(t)+s_{i}(t)\right), s_{i}(t)\right\rangle \\
= & -\left\langle F^{\prime}\left(z_{i}(t)+s_{i}(t)\right)-F^{\prime}\left(u(t)+s_{i}(t)\right), s_{i}(t)\right\rangle-\left\langle F^{\prime}\left(u(t)+s_{i}(t)\right), s_{i}(t)\right\rangle \\
= & -\sum_{j=1}^{m}\left\langle F^{\prime}\left(\phi_{j}^{i}(t)+s_{i}(t)\right)-F^{\prime}\left(\phi_{j-1}^{i}(t)+s_{i}(t)\right), s_{i}(t)\right\rangle \\
& -\left\langle F^{\prime}\left(u(t)+s_{i}(t)\right), s_{i}(t)\right\rangle .
\end{aligned}
$$


If $t \notin T^{i}$, then $s_{i}(t)=0$ and the above inequality holds trivially. Combining the above inequality with (77) and (9) and (20), we obtain that

$$
\begin{aligned}
& \sum_{k=1}^{c_{t}}\left(F\left(u(t)+e_{k}(t)\right)-F(u(t))\right) \\
& \left.\quad \leq \sum_{k=1}^{c_{t}} \sum_{i \in I(k)}\left\langle F^{\prime}(u(t))+s_{i}(t)\right), s_{i}(t)\right\rangle-\frac{\sigma}{2} \sum_{k=1}^{c_{t}} \sum_{i \in I(k)}\left\|s_{i}(t)\right\|^{2} \\
& \left.\quad=\sum_{i=1}^{m}\left\langle F^{\prime}(u(t))+s_{i}(t)\right), s_{i}(t)\right\rangle-\frac{\sigma}{2} \sum_{i=1}^{m}\left\|s_{i}(t)\right\|^{2} \\
& \quad \leq-\sum_{i=1}^{m} \sum_{j=1}^{m}\left\langle F^{\prime}\left(\phi_{j}^{i}(t)+s_{i}(t)\right)-F^{\prime}\left(\phi_{j-1}^{i}(t)+s_{i}(t)\right), s_{i}(t)\right\rangle-\frac{\sigma}{2} \sum_{i=1}^{m}\left\|s_{i}(t)\right\|^{2} .
\end{aligned}
$$

Substituting (21) into (19) and using (6) yields

$$
\begin{aligned}
F(u(t+1)) \leq & F(u(t)) \\
& +\gamma C_{2}\left(\sum_{j=1}^{m} \max _{i=1, \ldots, m}\left\|u_{j}\left(\tau_{j}^{i}(t)\right)-u_{j}(t)\right\|^{2}\right)^{\frac{1}{2}}\left(\sum_{i=1}^{m}\left\|s_{i}(t)\right\|^{2}\right)^{\frac{1}{2}} \\
& -\gamma \frac{\sigma}{2} \sum_{i=1}^{m}\left\|s_{i}(t)\right\|^{2} .
\end{aligned}
$$

Since $t-B+1 \leq \tau_{j}^{i}(t) \leq t$ for all $i$ and $j$, we also have from (10) and the triangle inequality that

$$
\left\|u_{j}\left(\tau_{j}^{i}(t)\right)-u_{j}(t)\right\|^{2} \leq \gamma^{2}\left(\sum_{\tau=t-B+1}^{t-1}\left\|s_{j}(\tau)\right\|\right)^{2} \leq \gamma^{2} B \sum_{\tau=t-B+1}^{t-1}\left\|s_{j}(\tau)\right\|^{2} .
$$

Combining (22) and (23) yields

$$
\begin{aligned}
F(u(t+1)) \leq & F(u(t)) \\
& +\gamma^{2} C_{2} \sqrt{B}\left(\sum_{j=1}^{m} \sum_{\tau=t-B+1}^{t-1}\left\|s_{j}(\tau)\right\|^{2}\right)^{\frac{1}{2}}\left(\sum_{i=1}^{m}\left\|s_{i}(t)\right\|^{2}\right)^{\frac{1}{2}} \\
& -\gamma \frac{\sigma}{2} \sum_{i=1}^{m}\left\|s_{i}(t)\right\|^{2} \\
\leq & F(u(t))+\gamma^{3} \frac{C_{2}^{2} B}{\sigma} \sum_{j=1}^{m} \sum_{\tau=t-B+1}^{t-1}\left\|s_{j}(\tau)\right\|^{2}-\gamma \frac{\sigma}{4} \sum_{i=1}^{m}\left\|s_{i}(t)\right\|^{2},
\end{aligned}
$$

where the second inequality uses the identity $a b \leq\left(a^{2}+b^{2}\right) / 2$ with $a$ and $b$ being the two square-root terms multiplied and divided, respectively, by $B^{1 / 4} \sqrt{2 \gamma C_{2} / \sigma}$. Applying the above argument successively to $t, t+1, \ldots, t+B-1$, we obtain

$$
\begin{aligned}
& F(u(t+B))-F(u(t)) \\
& \quad \leq-\gamma\left(\frac{\sigma}{4}-\frac{\gamma^{2} C_{2}^{2} B^{2}}{\sigma}\right) \sum_{j=1}^{m} \sum_{\tau=t}^{t+B-1}\left\|s_{j}(\tau)\right\|^{2}+\gamma^{3} \frac{C_{2}^{2} B^{2}}{\sigma} \sum_{j=1}^{m} \sum_{\tau=t-B+1}^{t-1}\left\|s_{j}(\tau)\right\|^{2} .
\end{aligned}
$$

This proves the lemma. 
The next key lemma estimates the optimality gap $F(u(t+B))-F(\bar{u})$, where $\bar{u}$ is the unique solution of (2).

Lemma 2 (Optimality gap estimate). Let $A_{3}$ and $A_{4}$ be defined by

$$
A_{4}=\frac{C_{2} B^{2}}{2}+\frac{8 C_{1}^{2} C_{2}^{2} B}{\sigma}, \quad A_{3}=\frac{3 C_{2}}{2}+\frac{6 C_{1}^{2} C_{2}^{2}}{\sigma}+A_{4} .
$$

For $t=0,1, \ldots$, we have

$$
\begin{aligned}
F(u(t+B))-F(\bar{u}) \leq & (1-\gamma)(F(u(t))-F(\bar{u})) \\
+ & \gamma A_{3} \sum_{j=1}^{m} \sum_{\tau=t}^{t+B-1}\left\|s_{j}(\tau)\right\|^{2}+\gamma^{3} A_{4} \sum_{j=1}^{m} \sum_{\tau=t-B+1}^{t-1}\left\|s_{j}(\tau)\right\|^{2}
\end{aligned}
$$

Proof. Fix any $t \in\{0,1, \ldots\}$. For each $i \in\{1, \ldots, m\}$, let $t^{i}$ denote the greatest element of $T^{i}$ less than $t+B$. Then we have from (11) and (17) that

$$
\left\langle F^{\prime}\left(z_{i}\left(t^{i}\right)+s_{i}\left(t^{i}\right)\right), v_{i}-w_{i}\left(t^{i}\right)\right\rangle \geq 0, \quad \forall v_{i} \in K_{i}
$$

We also have from (10) and (16) that

$$
\begin{aligned}
u_{i}(t+B) & =u_{i}\left(t^{i}\right)+\gamma s_{i}\left(t^{i}\right) \\
u(t+B) & =\sum_{i=1}^{m} u_{i}\left(t^{i}+1\right)=\sum_{i=1}^{m} u_{i}\left(t^{i}\right)+\gamma \sum_{i=1}^{m} s_{i}\left(t^{i}\right) .
\end{aligned}
$$

For notational simplicity, define

$$
w(t)=\sum_{i=1}^{m} w_{i}\left(t^{i}\right), \quad \hat{u}(t)=\sum_{i=1}^{m} u_{i}\left(t^{i}\right) .
$$

By assumption, there exists $\bar{u}_{i} \in K_{i}, i=1, \ldots, m$, such that (5) holds with $v_{i}=$ $w_{i}\left(t^{i}\right)$, i.e.,

$$
\bar{u}=\sum_{i=1}^{m} \bar{u}_{i} \quad \text { and } \quad\left(\sum_{i=1}^{m}\left\|w_{i}\left(t^{i}\right)-\bar{u}_{i}\right\|^{2}\right)^{\frac{1}{2}} \leq C_{1}\|w(t)-\bar{u}\| .
$$

Then $\left(\bar{u}_{1}, \ldots, \bar{u}_{m}\right)$ is a solution of the convex program (1) and, by $F$ being Gâteaudifferentiable, it satisfies the optimality condition

$$
\sum_{i=1}^{m}\left\langle F^{\prime}(\bar{u}), v_{i}-\bar{u}_{i}\right\rangle \geq 0, \quad \forall v_{i} \in K_{i}, i=1, \ldots, m .
$$

Defining

$$
\phi_{j}^{i}(t)=\sum_{k=1}^{j} w_{k}\left(t^{k}\right)+\sum_{k=j+1}^{m} u_{k}\left(\tau_{k}^{i}\left(t^{i}\right)\right), \quad j=0,1, \ldots, m
$$

we have that $\phi_{0}^{i}(t)=z_{i}\left(t^{i}\right)$ and $\phi_{m}^{i}(t)=w(t)$ and

$$
\phi_{j}^{i}(t)-\phi_{j-1}^{i}(t)=w_{j}\left(t^{j}\right)-u_{j}\left(\tau_{j}^{i}\left(t^{i}\right)\right) \in K_{j}^{\ominus}, \quad j=1, \ldots, m .
$$


Setting $v_{i}=\bar{u}_{i}$ in (26) and $v_{i}=w_{i}\left(t^{i}\right)$ in (28), we obtain that

$$
\begin{aligned}
&\left\langle F^{\prime}(w(t))-F^{\prime}(\bar{u}), w(t)-\bar{u}\right\rangle \leq\left\langle F^{\prime}(w(t)), w(t)-\bar{u}\right\rangle \\
& \leq \sum_{i=1}^{m}\left\langle F^{\prime}(w(t))-F^{\prime}\left(z_{i}\left(t^{i}\right)+s_{i}\left(t^{i}\right)\right), w_{i}\left(t^{i}\right)-\bar{u}_{i}\right\rangle \\
&= \sum_{i=1}^{m}\left\langle F^{\prime}(w(t))-F^{\prime}\left(z_{i}\left(t^{i}\right)\right), w_{i}\left(t^{i}\right)-\bar{u}_{i}\right\rangle \\
&+\sum_{i=1}^{m}\left\langle F^{\prime}\left(z_{i}\left(t^{i}\right)\right)-F^{\prime}\left(z_{i}\left(t^{i}\right)+s_{i}\left(t^{i}\right)\right), w_{i}\left(t^{i}\right)-\bar{u}_{i}\right\rangle \\
&= \sum_{i=1}^{m} \sum_{j=1}^{m}\left\langle F^{\prime}\left(\phi_{j}^{i}(t)\right)-F^{\prime}\left(\phi_{j-1}^{i}(t)\right), w_{i}\left(t^{i}\right)-\bar{u}_{i}\right\rangle \\
&+\sum_{i=1}^{m}\left\langle F^{\prime}\left(z_{i}\left(t^{i}\right)\right)-F^{\prime}\left(z_{i}\left(t^{i}\right)+s_{i}\left(t^{i}\right)\right), w_{i}\left(t^{i}\right)-\bar{u}_{i}\right\rangle \\
& \leq C_{2}\left(\sum_{j=1}^{m} \max _{i=1, \ldots, m}\left\|u_{j}\left(\tau_{j}^{i}\left(t^{i}\right)\right)-w_{j}\left(t^{j}\right)\right\|^{2}\right)^{\frac{1}{2}}\left(\sum_{i=1}^{m}\left\|w_{i}\left(t^{i}\right)-\bar{u}_{i}\right\|^{2}\right)^{\frac{1}{2}} \\
&+C_{2}\left(\sum_{i=1}^{m}\left\|s_{i}\left(t^{i}\right)\right\|^{2}\right)^{\frac{1}{2}}\left(\sum_{i=1}^{m}\left\|w_{i}\left(t^{i}\right)-\bar{u}_{i}\right\|^{2}\right)^{\frac{1}{2}} \\
& \leq C_{1} C_{2}\left(\sum_{j=1}^{m}\left(4 \gamma^{2} B \sum_{\tau=t-B+1}^{t+B-2}\left\|s_{j}(\tau)\right\|^{2}+2\left\|s_{j}\left(t^{j}\right)\right\|^{2}\right)\right)^{\frac{1}{2}}\|w(t)-\bar{u}\| \\
&+C_{1} C_{2}\left(\sum_{i=1}^{m}\left\|s_{i}\left(t^{i}\right)\right\|^{2}\right)^{\frac{1}{2}}\|w(t)-\bar{u}\|,
\end{aligned}
$$

where the third inequality uses (6) and (29); the fourth inequality uses (27) and the fact that

$$
\begin{aligned}
\left\|u_{j}\left(\tau_{j}^{i}\left(t^{i}\right)\right)-w_{j}\left(t^{j}\right)\right\|^{2} & =\left\|u_{j}\left(\tau_{j}^{i}\left(t^{i}\right)\right)-u_{j}\left(t^{j}\right)-s_{j}\left(t^{j}\right)\right\|^{2} \\
& \leq 2\left\|u_{j}\left(\tau_{j}^{i}\left(t^{i}\right)\right)-u_{j}\left(t^{j}\right)\right\|^{2}+2\left\|s_{j}\left(t^{j}\right)\right\|^{2} \\
& \leq 2 \gamma^{2}\left(\sum_{\tau=t-B+1}^{t+B-2}\left\|s_{j}(\tau)\right\|\right)^{2}+2\left\|s_{j}\left(t^{j}\right)\right\|^{2} \\
& \leq 4 \gamma^{2} B \sum_{\tau=t-B+1}^{t+B-2}\left\|s_{j}(\tau)\right\|^{2}+2\left\|s_{j}\left(t^{j}\right)\right\|^{2}
\end{aligned}
$$

(see (10), (11), (13), (14)). Also, the strong monotonicity (3) of $F^{\prime}$ on $K$ implies

$$
\left\langle F^{\prime}(w(t))-F^{\prime}(\bar{u}), w(t)-\bar{u}\right\rangle \geq \sigma\|w(t)-\bar{u}\|^{2}
$$


which together with (30) yields

$$
\begin{aligned}
\|w(t)-\bar{u}\| & \leq \frac{C_{1} C_{2}}{\sigma}\left(\sum_{j=1}^{m}\left(4 \gamma^{2} B \sum_{\tau=t-B+1}^{t+B-2}\left\|s_{j}(\tau)\right\|^{2}+2\left\|s_{j}\left(t^{j}\right)\right\|^{2}\right)\right)^{\frac{1}{2}} \\
& +\frac{C_{1} C_{2}}{\sigma}\left(\sum_{i=1}^{m}\left\|s_{i}\left(t^{i}\right)\right\|^{2}\right)^{\frac{1}{2}}
\end{aligned}
$$

Next, since $F^{\prime}(w(t))$ is a subgradient of $F$ at $w(t)$ [20, p. 23], we have

$$
F(w(t))-F(\bar{u}) \leq\left\langle F^{\prime}(w(t)), w(t)-\bar{u}\right\rangle,
$$

so putting $v_{i}=\bar{u}_{i}$ in (26) and adding it to the above inequality yields

$$
\begin{gathered}
F(w(t))-F(\bar{u}) \\
\leq \sum_{i=1}^{m}\left\langle F^{\prime}(w(t))-F^{\prime}\left(z_{i}\left(t^{i}\right)+s_{i}\left(t^{i}\right)\right), w_{i}\left(t^{i}\right)-\bar{u}_{i}\right\rangle \\
\leq \frac{C_{1}^{2} C_{2}^{2}}{\sigma}\left(\left(\sum_{j=1}^{m}\left(4 \gamma^{2} B \sum_{\tau=t-B+1}^{t+B-2}\left\|s_{j}(\tau)\right\|^{2}+2\left\|s_{j}\left(t^{j}\right)\right\|^{2}\right)\right)^{\frac{1}{2}}\right. \\
\left.+\left(\sum_{i=1}^{m}\left\|s_{i}\left(t^{i}\right)\right\|^{2}\right)^{\frac{1}{2}}\right)^{2} \\
\leq \frac{2 C_{1}^{2} C_{2}^{2}}{\sigma}\left(4 \gamma^{2} B \sum_{j=1}^{m} \sum_{\tau=t-B+1}^{t+B-2}\left\|s_{j}(\tau)\right\|^{2}+3 \sum_{i=1}^{m}\left\|s_{i}\left(t^{i}\right)\right\|^{2}\right),
\end{gathered}
$$

where the second inequality uses (30) and (31) and the last inequality follows from the identity $(a+b)^{2} \leq 2\left(a^{2}+b^{2}\right)$.

Next we estimate $F(\hat{u}(t))-F(u(t))$. Let $\bar{t}=\max _{i=1, \ldots, m} t^{i}$ and, for each $i \in$ $\{1, \ldots, m\}$ and $\tau \in\{t, \ldots, \bar{t}\}$, define

$$
\tilde{u}_{i}(\tau)=u_{i}\left(\min \left\{\tau, t^{i}\right\}\right), \quad \tilde{u}(\tau)=\sum_{i=1}^{m} \tilde{u}_{i}(\tau)
$$

Then, for each $i \in\{1, \ldots, m\}$ and $\tau \in\{t, \ldots, \bar{t}-1\}$, either $\tilde{u}_{i}(\tau+1)=\tilde{u}_{i}(\tau)$ so that

$$
\left\langle F^{\prime}\left(z_{i}(\tau)+s_{i}(\tau)\right), \tilde{u}_{i}(\tau)-\tilde{u}_{i}(\tau+1)\right\rangle=0
$$

or $\tilde{u}_{i}(\tau+1) \neq \tilde{u}_{i}(\tau)$ so that $\tau \in T^{i}$ and $\tau<t^{i}$, implying by (11) and (17) that

$$
\left\langle F^{\prime}\left(z_{i}(\tau)+s_{i}(\tau)\right), u_{i}(\tau)-w_{i}(\tau)\right\rangle \geq 0
$$

and hence, by (33), that

$$
\begin{aligned}
\left\langle F^{\prime}\left(z_{i}(\tau)+s_{i}(\tau)\right), \tilde{u}_{i}(\tau)-\tilde{u}_{i}(\tau+1)\right\rangle & =\left\langle F^{\prime}\left(z_{i}(\tau)+s_{i}(\tau)\right), u_{i}(\tau)-u_{i}(\tau+1)\right\rangle \\
& =\gamma\left\langle F^{\prime}\left(z_{i}(\tau)+s_{i}(\tau)\right), u_{i}(\tau)-w_{i}(\tau)\right\rangle \geq 0 .
\end{aligned}
$$

Using this and defining

$$
\phi_{j}^{i}(\tau)=\sum_{k=1}^{j} \tilde{u}_{k}(\tau+1)+\sum_{k=j+1}^{m} u_{k}\left(\tau_{k}^{i}(\tau)\right), \quad j=0,1, \ldots, m,
$$


we obtain that

$$
\begin{aligned}
& F(\tilde{u}(\tau+1))-F(\tilde{u}(\tau)) \\
& \leq-\left\langle F^{\prime}(\tilde{u}(\tau+1)), \tilde{u}(\tau)-\tilde{u}(\tau+1)\right\rangle \\
& \leq \sum_{i=1}^{m}\left\langle F^{\prime}\left(z_{i}(\tau)+s_{i}(\tau)\right)-F^{\prime}(\tilde{u}(\tau+1)), \tilde{u}_{i}(\tau)-\tilde{u}_{i}(\tau+1)\right\rangle \\
& =\sum_{i=1}^{m}\left\langle F^{\prime}\left(z_{i}(\tau)\right)-F^{\prime}(\tilde{u}(\tau+1)), \tilde{u}_{i}(\tau)-\tilde{u}_{i}(\tau+1)\right\rangle \\
& +\sum_{i=1}^{m}\left\langle F^{\prime}\left(z_{i}(\tau)+s_{i}(\tau)\right)-F^{\prime}\left(z_{i}(\tau)\right), \tilde{u}_{i}(\tau)-\tilde{u}_{i}(\tau+1)\right\rangle \\
& =\sum_{i=1}^{m} \sum_{j=1}^{m}\left\langle F^{\prime}\left(\phi_{j-1}^{i}(\tau)\right)-F^{\prime}\left(\phi_{j}^{i}(\tau)\right), \tilde{u}_{i}(\tau)-\tilde{u}_{i}(\tau+1)\right\rangle \\
& +\sum_{i=1}^{m}\left\langle F^{\prime}\left(z_{i}(\tau)+s_{i}(\tau)\right)-F^{\prime}\left(z_{i}(\tau)\right), \tilde{u}_{i}(\tau)-\tilde{u}_{i}(\tau+1)\right\rangle \\
& \leq C_{2}\left(\sum_{j=1}^{m} \max _{i=1, \ldots, m}\left\|\phi_{j-1}^{i}(\tau)-\phi_{j}^{i}(\tau)\right\|^{2}\right)^{\frac{1}{2}}\left(\sum_{i=1}^{m}\left\|\tilde{u}_{i}(\tau)-\tilde{u}_{i}(\tau+1)\right\|^{2}\right)^{\frac{1}{2}} \\
& +C_{2}\left(\max _{i=1, \ldots, m}\left\|s_{i}(\tau)\right\|^{2}\right)^{\frac{1}{2}}\left(\sum_{i=1}^{m}\left\|\tilde{u}_{i}(\tau)-\tilde{u}_{i}(\tau+1)\right\|^{2}\right)^{\frac{1}{2}} \\
& \leq \gamma C_{2}\left(\sum_{j=1}^{m}\left(\max _{i=1, \ldots, m}\left\|\tilde{u}_{j}(\tau+1)-u_{j}\left(\tau_{j}^{i}(\tau)\right)\right\|^{2}\right)\right)^{\frac{1}{2}}\left(\sum_{i=1}^{m}\left\|s_{i}(\tau)\right\|^{2}\right)^{\frac{1}{2}} \\
& +\gamma C_{2} \sum_{i=1}^{m}\left\|s_{i}(\tau)\right\|^{2} \\
& \leq \gamma C_{2}\left(\gamma^{2} B \sum_{j=1}^{m} \sum_{\nu=\tau-B+1}^{\tau+1}\left\|s_{j}(\nu)\right\|^{2}\right)^{\frac{1}{2}}\left(\sum_{i=1}^{m}\left\|s_{i}(\tau)\right\|^{2}\right)^{\frac{1}{2}}+\gamma C_{2} \sum_{i=1}^{m}\left\|s_{i}(\tau)\right\|^{2} \\
& \leq \gamma^{3} \frac{C_{2} B}{2} \sum_{j=1}^{m} \sum_{\nu=\tau-B+1}^{\tau+1}\left\|s_{j}(\nu)\right\|^{2}+\gamma \frac{3 C_{2}}{2} \sum_{i=1}^{m}\left\|s_{i}(\tau)\right\|^{2},
\end{aligned}
$$

where the first inequality uses the subgradient property of $F^{\prime}(\tilde{u}(\tau+1))$ 20, p. 23]; the third inequality uses (6); the fourth and fifth inequalities use (33) and (10) and an inequality analogous to (23); the last inequality uses the identity $a b \leq\left(a^{2}+b^{2}\right) / 2$ with $a$ and $b$ being the two square-root terms. Summing the above inequality over $\tau=t, t+1, \ldots, \bar{t}-1$ and observing that $\tilde{u}(\bar{t})=\hat{u}(t)$ and $\tilde{u}(t)=u(t)$, we then have

$$
\begin{aligned}
F(\hat{u}(t))-F(u(t)) & \leq \gamma^{3} \frac{C_{2} B}{2} \sum_{j=1}^{m} \sum_{\tau=t}^{\bar{t}-1} \sum_{\nu=\tau-B+1}^{\tau+1}\left\|s_{j}(\nu)\right\|^{2}+\gamma \frac{3 C_{2}}{2} \sum_{i=1}^{m} \sum_{\tau=t}^{t-1}\left\|s_{i}(\tau)\right\|^{2} \\
& \leq \gamma^{3} \frac{C_{2} B^{2}}{2} \sum_{j=1}^{m} \sum_{\tau=t-B+1}^{t+B-1}\left\|s_{j}(\tau)\right\|^{2}+\gamma \frac{3 C_{2}}{2} \sum_{i=1}^{m} \sum_{\tau=t}^{t+B-1}\left\|s_{i}(\tau)\right\|^{2} .
\end{aligned}
$$


Finally, using the convexity of $F$ and $\gamma \in[0,1]$, we see from (11) and (32) and (35) that

$$
\begin{aligned}
F(u(t+B))-F(\bar{u}) & F\left(\sum_{i=1}^{m} u_{i}(t+B)\right)-F(\bar{u}) \\
= & F\left(\sum_{i=1}^{m}\left(u_{i}\left(t^{i}\right)+\gamma\left(w_{i}\left(t^{i}\right)-u_{i}\left(t^{i}\right)\right)\right)\right)-F(\bar{u}) \\
= & F((1-\gamma) \hat{u}(t)+\gamma w(t))-F(\bar{u}) \\
\leq & (1-\gamma) F(\hat{u}(t))+\gamma F(w(t))-F(\bar{u}) \\
= & (1-\gamma)(F(\hat{u}(t))-F(\bar{u}))+\gamma(F(w(t))-F(\bar{u})) \\
\leq & (1-\gamma)(F(u(t))-F(\bar{u})) \\
& +\gamma^{3} \frac{C_{2} B^{2}}{2} \sum_{j=1}^{m} \sum_{\tau=t-B+1}^{t+B-1}\left\|s_{j}(\tau)\right\|^{2}+\gamma \frac{3 C_{2}}{2} \sum_{i=1}^{m} \sum_{\tau=t}^{t+B-1}\left\|s_{i}(\tau)\right\|^{2} \\
& +\gamma^{3} \frac{8 C_{1}^{2} C_{2}^{2} B}{\sigma} \sum_{j=1}^{m} \sum_{\tau=t-B+1}^{t+B-2}\left\|s_{j}(\tau)\right\|^{2}+\gamma \frac{6 C_{1}^{2} C_{2}^{2}}{\sigma} \sum_{i=1}^{m}\left\|s_{i}\left(t^{i}\right)\right\|^{2} .
\end{aligned}
$$

Using $\gamma \leq 1$ then proves the lemma.

We will now use Lemmas 1 and 2 to prove our convergence rate result. To simplify the notations, define

$$
a_{k}=F(u(k B))-F(\bar{u}), \quad b_{k}=\sum_{j=1}^{m} \sum_{\tau=k B-B}^{k B-1}\left\|s_{j}(\tau)\right\|^{2}, \quad k=1,2, \ldots
$$

By Lemmas 1 and 2, we have

$$
\begin{array}{r}
a_{k} \leq a_{k-1}-\gamma A_{1} b_{k}+\gamma^{3} A_{2} b_{k-1}, \\
a_{k} \leq(1-\gamma) a_{k-1}+\gamma A_{3} b_{k}+\gamma^{3} A_{4} b_{k-1},
\end{array}
$$

where $A_{1}, A_{2}, A_{3}, A_{4}$ are given by (18) and (25). By (15), we have $A_{1}>0$. Choose $\gamma$ sufficiently small so that

$$
\begin{aligned}
\varrho & =\max \left\{\left(1+\frac{A_{1}}{A_{3}}\right)^{-1}\left(1+(1-\gamma) \frac{A_{1}}{A_{3}}+\gamma^{3 / 2}\left(A_{2}+\frac{A_{1} A_{4}}{A_{3}}\right)\right), A_{1}^{-1}\left(\gamma^{1 / 2}+\gamma^{2} A_{2}\right)\right\} \\
& <1 .
\end{aligned}
$$

Also, define $a=\max \left\{a_{1}, \gamma^{3 / 2} b_{1}\right\} / \varrho$. We claim that

$$
\max \left\{a_{n}, \gamma^{3 / 2} b_{n}\right\} \leq a \varrho^{n}
$$

for $n=1,2, \ldots$. We prove this by induction on $n$. Clearly (39) holds for $n=1$ by our definition of $a$. Suppose (39) holds for $n=k-1$, where $k>1$. Multiplying (37) by $A_{1} / A_{3}$ and adding it to (36) gives

$$
\left(1+\frac{A_{1}}{A_{3}}\right) a_{k} \leq\left(1+(1-\gamma) \frac{A_{1}}{A_{3}}\right) a_{k-1}+\gamma^{3 / 2}\left(A_{2}+\frac{A_{1} A_{4}}{A_{3}}\right)\left(\gamma^{3 / 2} b_{k-1}\right),
$$


which together with the inductive hypothesis $\max \left\{a_{k-1}, \gamma^{3 / 2} b_{k-1}\right\} \leq a \varrho^{k-1}$ and (38) yields

$$
a_{k} \leq\left(1+\frac{A_{1}}{A_{3}}\right)^{-1}\left(1+(1-\gamma) \frac{A_{1}}{A_{3}}+\gamma^{3 / 2}\left(A_{2}+\frac{A_{1} A_{4}}{A_{3}}\right)\right) a \varrho^{k-1} \leq a \varrho^{k} .
$$

Similarly, (36) and $a_{k} \geq 0$ give

$$
\gamma^{3 / 2} A_{1} b_{k} \leq \gamma^{1 / 2} a_{k-1}+\gamma^{2} A_{2}\left(\gamma^{3 / 2} b_{k-1}\right),
$$

which together with $\max \left\{a_{k-1}, \gamma^{3 / 2} b_{k-1}\right\} \leq a \varrho^{k-1}$ and (38) yields

$$
\gamma^{3 / 2} b_{k} \leq A_{1}^{-1}\left(\gamma^{1 / 2}+\gamma^{2} A_{2}\right) a \varrho^{k-1} \leq a \varrho^{k} .
$$

This shows that (39) holds for $n=k$, completing our induction proof.

Thus, we have shown linear rate of convergence (in the root sense) for both $a_{n}$ and $b_{n}$, with a factor of $\varrho$. The latter implies $u_{i}(t), t=0,1, \ldots$, is a Cauchy sequence for each $i$ and hence it converges strongly. This is summarized in the theorem below.

Theorem 1. Consider the minimization problem (2) and the space decomposition (4) of Section 国 (see (3), (5) -(9)). Let $\left(u_{1}(t), \ldots, u_{m}(t)\right), t=0,1, \ldots$, be generated by the asynchronous space decomposition method of Section 3 (see (10)-(12) and (13), (14)) with stepsize $\gamma$ satisfying (15), (38). Then, there exist $a>0$ and $\varrho \in$ $(0,1)$, depending on $\sigma, C_{1}, C_{2}$ and $B, \gamma$ only, such that

$$
F(u(n B))-F(\bar{u}) \leq a \varrho^{n}, \quad n=1,2, \ldots,
$$

where $u(t)$ is given by (16) and $\bar{u}$ denotes the unique solution of (2). Moreover, $u(t)$ converges strongly to $\bar{u}$ and, for each $i \in\{1, \ldots, m\}, u_{i}(t)$ converges strongly as $t \rightarrow \infty$.

\section{Convergence RAte}

\section{OF THE SYNCHRONOUS SEQUENTIAL AND PARALLEL ALGORITHMS}

It is readily seen that the following Jacobi version of the method is a special case of the asynchronous space decomposition method (10)-(12) with $T^{i}=\{0,1, \ldots\}$ and $\tau_{j}^{i}(t)=t$ for all $i, j, t$ (so $B=1$ and $c_{t}=c$ ). Thus, Theorem 1 can be applied to establish its linear convergence and obtain an estimate of the factor $\varrho$ under the assumptions of Section 2, Moreover, by observing that in this case the left-hand side of (23) is zero so that Lemma 1 1 holds with $A_{2}=0$, the stepsize restriction (15) can be relaxed to $\gamma \leq 1 / c_{t}$.

\section{Algorithm 1.}

Step 1. Choose initial values $u_{i}(0) \in K_{i}, i=1, \ldots, m$, and stepsize $\gamma=1 / c$, where $c$ is defined as in Section Q.

Step 2. For each $t=0,1, \ldots$, find $w_{i}(t) \in K_{i}$ in parallel for $i=1, \ldots, m$ that satisfies

$$
F\left(\sum_{j \neq i} u_{j}(t)+w_{i}(t)\right) \leq F\left(\sum_{j \neq i} u_{j}(t)+v_{i}\right), \quad \forall v_{i} \in K_{i} .
$$

Step 3. Set

$$
u_{i}(t+1)=u_{i}(t)+\gamma\left(w_{i}(t)-u_{i}(t)\right),
$$

and go to the next iteration. 
The following Gauss-Seidel version of the method is also a special case of the asynchronous space decomposition method (10)-(12) with $\gamma=1, T^{i}=$ $\{i-1+k m\}_{k=0,1, \ldots}$ and $\tau_{j}^{i}(t)=t$ for all $i, j, t$ (so $B=m$ and $c_{t}=1$ ), Here Theorem 1 cannot be directly applied due to $\gamma=1$ possibly violating (15). However, by observing that in this case the left-hand side of (23) is again zero so that Lemma 1 holds with $A_{2}=0$, the proof of the theorem can be easily modified to establish linear convergence of this method under the assumptions of Section 2 with factor $\varrho$ depending on $m, \sigma, C_{1}, C_{2}$ only. Moreover, by grouping sets of the same color into one set, we can ensure that $m=c$, where $c$ is defined as in Section 2

\section{Algorithm 2.}

Step 1. Choose initial values $u_{i}(0) \in K_{i}, i=1, \ldots, m$.

Step 2. For each $t=0,1, \ldots$, find $u_{i}(t+1) \in K_{i}$ sequentially for $i=1, \ldots, m$ that satisfies

$$
\begin{aligned}
& F\left(\sum_{j<i} u_{j}(t+1)+u_{i}(t+1)+\sum_{j>i} u_{j}(t)\right) \\
& \quad \leq F\left(\sum_{j<i} u_{j}(t+1)+v_{i}+\sum_{j>i} u_{j}(t)\right), \quad \forall v_{i} \in K_{i} .
\end{aligned}
$$

Step 3. Go to the next iteration.

The above two methods for solving (2) were studied in [47] (also see [48, 49, 50]), where convergence of the methods was proved under weaker assumptions. However, no rate of convergence result was given. In [52], a linear rate of convergence for the above two methods was proved for the unconstrained case of $K=V$. In the finite-dimensional case of $K=V=\Re^{n}$, the literature concerning the linear rate of convergence is very rich. However, the study for linear convergence rate for general convex sets $K_{i}$ is very sparse. The linear rate of convergence for the Gauss-Seidel method for general convex sets $K_{i}$ can also be inferred from the results in [34, 35. and references therein, but our estimate of the convergence factor is new.

In 52], the minimization subproblem at each iteration is solved inexactly. We can do likewise in the constrained case. In particular, the proof of Theorem 1 (see (21) and (26)) suggests that the exact minimization condition (17) can be relaxed to the inexact minimization condition

$$
\left\langle F^{\prime}\left(z_{i}(t)+w_{i}(t)-u_{i}(t)\right), v_{i}-w_{i}(t)\right\rangle \geq-\frac{\sigma_{0}}{2}\left\|w_{i}(t)-u_{i}(t)\right\|^{2}, \quad \forall v_{i} \in K_{i},
$$

with $0<\sigma_{0}<\sigma$. However, $\sigma$ would need to be known explicitly and both $\gamma$ and $\varrho$ would depend on $\sigma_{0}$.

\section{Applichtions to convex programming}

In this section we consider the Euclidean space $V=V^{\prime}=\Re^{n}$, which is the space of $n$-dimensional real column vectors with duality pairing $\langle f, x\rangle=f^{T} x$ and norm $\|x\|=\sqrt{x^{T} x}$, where $x^{T}$ denotes transpose of $x$. We will discuss choices of the space decomposition (44) and the corresponding estimates for $C_{1}, C_{2}, c$ in (5), (6), (7). In the case of nonlinear network flow, we will also relate our asynchronous method to those studied in [5, \$7.2.3], [56]. 
6.1. Primal applications. Consider the problem (2), where $F: \Re^{n} \mapsto \Re$ is a differentiable convex function and $K$ is a nonempty polyhedral set in $\Re^{n}$. Then $F$ is continuous [42, p. 82] and continuously differentiable [42, p. 246]. We assume that the gradient $F^{\prime}=\left(\frac{\partial F}{\partial x_{j}}\right)_{j=1}^{n}$ is strongly monotone and Lipschitz continuous on $K$, and we choose a space decomposition (4) such that each $K_{i}$ is a polyhedral set.

Since each $K_{i}$ is a polyhedral set, a result of Hoffman on the Lipschitzian behavior of solutions of a linear system with respect to the right-hand side (see [13]) implies that, for any $v_{i} \in K_{i}, i=1, \ldots, m$, there exists $\bar{u}_{i} \in K_{i}$ satisfying (5), where the constant $C_{1}$ depends on $m$ and certain condition numbers for $K_{i}, i=1, \ldots, m$. In cases where each $K_{i}$ has a simple structure, such as the Cartesian product of closed intervals, $C_{1}$ may be estimated explicitly. For a coloring of the sets, if $K_{i}$ and $K_{j}$ are not orthogonal, i.e., $\left(v_{i}\right)^{T} v_{j} \neq 0$ for some $v_{i} \in K_{i}, v_{j} \in K_{j}$, then we paint them different colors. Let $\hat{c}$ be the maximum number of sets $K_{j}$ that are not orthogonal to an arbitrary set $K_{i}$. Then an analysis similar to that used in subsection [7.1.3 shows that (6) holds with $C_{2}=L \hat{c}$, where $L$ is the Lipschitz constant for $F^{\prime}$.

6.2. Dual applications. Consider the linearly constrained convex program

$$
\text { minimize } G(x) \text { subject to } A x \geq b,
$$

where $G: \Re^{n} \mapsto \Re$ is a strictly convex differentiable function, $b \in \Re^{m}$, and $A \in$ $\Re^{m \times n}$ has nonzero rows. We assume there exists $\tilde{x} \in \Re^{n}$ satisfying $A \tilde{x}=b$. By attaching Lagrange multipliers $\lambda \in \Re^{m}$ to the inequalities $A x \geq b$ in (40), we obtain the Lagrangian dual problem

$$
\min _{\lambda \in \Re_{+}^{m}} G^{*}\left(A^{T} \lambda\right)-b^{T} \lambda,
$$

where $\Re_{+}^{m}$ denotes the nonnegative orthant in $\Re^{m}$, and $G^{*}$ is the convex conjugate (also called Legendre-Fenchel transform) of $G$ defined by

$$
G^{*}(u)=\sup _{x \in \Re^{n}}\left\{u^{T} x-G(x)\right\}
$$

(see [24, 42]). The convex programs (40) and (41) are dual in the sense that one has a solution if and only if the other does, and these solutions satisfies $G^{\prime}(x)=A^{T} \lambda$ [42, Cor. 28.3.1 and 28.4.1]. Using $b=A \tilde{x}$, we can rewrite the dual problem (41) in the form of (2) with

$$
F(u)=G^{*}(u)-\tilde{x}^{T} u, \quad K=\left\{u \in \Re^{n}: u=A^{T} \lambda \text { for some } \lambda \in \Re_{+}^{m}\right\} .
$$

We assume that $\left(G^{*}\right)^{\prime}$ is strongly monotone and Lipschitz continuous on $\Re^{n}$, so that $F$ satisfies (3) for some $\sigma>0$. If $G$ is twice differentiable, this assumption essentially amounts to $G^{\prime \prime}$ having bounded eigenvalues and the Hessian $\left(G^{\prime \prime}\right)^{-1}$ having bounded entries on $\Re^{n}$. Let $\bar{u}$ denote the unique solution of (2) and let $A_{i}$ denote the $i$ th row of $A$.

We can decompose $K$ in the form (4) with

$$
K_{i}=\left\{u_{i} \in \Re^{n}: u_{i}=A_{i}^{T} \lambda_{i} \text { for some } \lambda_{i} \in \Re_{+}\right\} .
$$

First we show that, for any $v_{i} \in K_{i}, i=1, \ldots, m$, there exists $\bar{u}_{i} \in K_{i}$ satisfying (5), where

$$
C_{1}=\max _{\emptyset \neq I \subset\{1, \ldots, m\}}\left\|D_{I}^{-1} B_{I}\left(B_{I}^{T} D_{I}^{-2} B_{I}\right)^{-1}\right\|,
$$

with $D_{I}$ being the diagonal matrix with diagonal entries $\left\|A_{i}^{T}\right\|, i \in I$, and $B_{I}$ being a submatrix of $A_{I}=\left[A_{i}\right]_{i \in I}$ comprising linearly independent columns of 
$A_{I}$ spanning the column space of $A_{I}$. To see this, notice that $\bar{u}=A^{T} \bar{\lambda}$ for some $\bar{\lambda} \in \Re_{+}^{m}$ and $v_{i}=A_{i}^{T} \mu_{i}$ for some $\mu_{i} \in \Re_{+}$. Moreover, $u_{i} \in K_{i}, i=1, \ldots, m$, satisfies $\sum_{i=1}^{m} u_{i}=\bar{u}$ if and only if $u_{i}=A_{i}^{T} \lambda_{i}$ and $A^{T} \lambda=A^{T} \bar{\lambda}$ for some $\lambda=\left(\lambda_{i}\right)_{i=1}^{m} \in \Re_{+}^{m}$. Thus, minimizing $\sum_{i=1}^{m}\left\|u_{i}-v_{i}\right\|^{2}$ subject to $u_{i} \in K_{i}$ and $\sum_{i=1}^{m} u_{i}=\bar{u}$ is equivalent to minimizing

$$
\sum_{i=1}^{m}\left\|A_{i}^{T} \lambda_{i}-A_{i}^{T} \mu_{i}\right\|^{2}=\sum_{i=1}^{m}\left\|A_{i}^{T}\right\|^{2}\left|\lambda_{i}-\mu_{i}\right|^{2}=\|D(\lambda-\mu)\|^{2}
$$

subject to $A^{T} \lambda=A^{T} \bar{\lambda}, \lambda \geq 0$, where $\mu=\left(\mu_{i}\right)_{i=1}^{m}$. By making the variable substitution $\xi=\lambda-\mu$ and letting $r=A^{T}(\bar{\lambda}-\mu)$, this in turn is equivalent to

$$
\text { minimize }\|D \xi\| \quad \text { subject to } A^{T} \xi=r, \xi \geq-\mu \text {. }
$$

Since $\mu \geq 0$, the optimal value of (43) equals zero if $r=0$. Suppose instead that $r \neq 0$. Since $\mu \geq 0$, the optimal value of (43) is below that of

$$
\text { minimize }\|D \xi\| \text { subject to } A^{T} \xi=r, \xi \geq 0 .
$$

The latter has a unique solution, which we denote by $\bar{\xi}=\left(\bar{\xi}_{i}\right)_{i=1}^{m}$. Since $r \neq 0$, then $\bar{\xi} \neq 0$. Let $I=\left\{i \in\{1, \ldots, m\}: \bar{\xi}_{i} \neq 0\right\}$. Then, $\bar{\xi}_{I}=\left(\bar{\xi}_{i}\right)_{i \in I}$ solves the reduced problem

$$
\text { minimize }\left\|D_{I} \xi_{I}\right\| \quad \text { subject to } \quad B_{I}^{T} \xi_{I}=r_{I},
$$

where $r_{I}$ is the subvector of $r$ corresponding to the columns of $B_{I}$. This yields $\bar{\xi}_{I}=D_{I}^{-2} B_{I}\left(B_{I}^{T} D_{I}^{-2} B_{I}\right)^{-1} r_{I}$, and hence

$$
\begin{aligned}
\text { optimal value of (43) } & \leq\left\|D_{I} \bar{\xi}_{I}\right\| \\
& =\left\|D_{I}^{-1} B_{I}\left(B_{I}^{T} D_{I}^{-2} B_{I}\right)^{-1} r_{I}\right\| \\
& \leq C_{1}\left\|r_{I}\right\| \\
& \leq C_{1}\|r\| \\
& =C_{1}\left\|A^{T}(\bar{\lambda}-\mu)\right\| \\
& =C_{1}\left\|\bar{u}-\sum_{i=1}^{m} v_{i}\right\| .
\end{aligned}
$$

Since $K_{i}$ and $K_{j}$ lie in orthogonal subspaces if $A_{i} A_{j}^{T}=0$, we can color $K_{1}, \ldots, K_{m}$ as discussed in subsection 6.1 and show that (6) holds with $C_{2}=L \hat{c}$, where $L$ is the Lipschitz constant for $\left(G^{*}\right)^{\prime}$ and $\hat{c}$ is the maximum number of rows $A_{j}$ that are not orthogonal to an arbitrary row $A_{i}$.

If we replace the inequality $A x \geq b$ in (40) by an equation $A x=b$, the constraint $\lambda \in \Re_{+}^{m}$ in (41) would be replaced accordingly by $\lambda \in \Re^{m}$ and it suffices to fix $I=\{1, \ldots, m\}$ in the estimate (42). This estimate further simplifies if $A$ has full row rank, in which case $B_{I}$ is square and invertible. If $A$ does not have full row rank, we could remove the redundant rows, but our experience with network flow problems suggests that this removal can slow the convergence of Gauss-Seidel methods on the problem [56].

In the case of a nonlinear network flow problem 43, where $A$ is the node-arc incidence matrix for a connected diagraph with $m$ nodes and $n$ arcs (i.e., every column of $A$ has one 1 and one -1 in two of its rows, and a 0 in the remaining rows), we can estimate $C_{1}$ explicitly in terms of $m$ and $n$ as follows: For any $v_{i} \in K_{i}$, $i=1, \ldots, m$, we have $\bar{u}=A^{T} \bar{\lambda}=\left(\bar{\lambda}_{k}-\bar{\lambda}_{l}\right)_{j=1 ; j \sim(k, l)}^{n}$ for some $\bar{\lambda}=\left(\bar{\lambda}_{i}\right)_{i=1}^{m} \in \Re_{+}^{m}$ 
and $v_{i}=A_{i}^{T} \mu_{i}$ for some $\mu_{i} \in \Re_{+}$, where $k \sim(i, j)$ means that column $k$ has a 1 in row $i$ and a -1 in row $j$ or, equivalently, arc $k$ is directed from node $i$ to node $j$. Choose any spanning tree for the diagraph and choose a node $\bar{i}$ such that $\bar{\lambda}_{\bar{i}}=\min _{i} \bar{\lambda}_{i}$. Let $\lambda_{i}=\mu_{\bar{i}}+\left(\bar{\lambda}_{i}-\bar{\lambda}_{\bar{i}}\right)$ and $u_{i}=A_{i}^{T} \lambda_{i}$ for all nodes $i$ in the network. Since $\mu_{\bar{i}} \geq 0$ and $\bar{\lambda}_{i} \geq \bar{\lambda}_{\bar{i}}$, we have $\lambda_{i} \geq 0$ for all nodes $i$. Since each node $i$ can be reached from $\bar{i}$ via a simple path $P_{i}$ in the spanning tree, we also have

$$
\begin{aligned}
\left|\lambda_{i}-\mu_{i}\right| & =\left|-\sum_{(k, l) \in P_{i}^{+}}\left(\lambda_{k}-\mu_{k}-\lambda_{l}+\mu_{l}\right)+\sum_{(k, l) \in P_{i}^{-}}\left(\lambda_{k}-\mu_{k}-\lambda_{l}+\mu_{l}\right)\right| \\
& =\left|-\sum_{(k, l) \in P_{i}^{+}}\left(\bar{\lambda}_{k}-\bar{\lambda}_{l}-\mu_{k}+\mu_{l}\right)+\sum_{(k, l) \in P_{i}^{-}}\left(\bar{\lambda}_{k}-\bar{\lambda}_{l}-\mu_{k}+\mu_{l}\right)\right| \\
& \leq \sum_{(k, l) \in P_{i}}\left|\bar{\lambda}_{k}-\bar{\lambda}_{l}-\mu_{k}+\mu_{l}\right| \\
& \leq \sqrt{h_{i}}\left(\sum_{(k, l) \in P_{i}}\left|\bar{\lambda}_{k}-\bar{\lambda}_{l}-\mu_{k}+\mu_{l}\right|^{2}\right)^{\frac{1}{2}} \\
& \leq \sqrt{h_{i}}\left(\sum_{j=1}^{n}\left|\bar{\lambda}_{k}-\bar{\lambda}_{l}-\mu_{k}+\mu_{l}\right|^{2}\right)^{\frac{1}{2}} \\
& =\sqrt{h_{i}}\left\|\sum_{j \sim(k, l)}^{m} \sum_{p=1}^{m} v_{p}\right\|,
\end{aligned}
$$

where $P_{i}^{+}$and $P_{i}^{-}$denote the set of forward arcs and backward $\operatorname{arcs}$ in $P_{i}$ and $h_{i}$ denotes the number of arcs in $P_{i}$. Thus,

$$
\begin{aligned}
\sum_{i=1}^{m}\left\|u_{i}-v_{i}\right\|^{2}=\sum_{i=1}^{m}\left\|A_{i}^{T}\left(\lambda_{i}-\mu_{i}\right)\right\|^{2} \\
=\sum_{i=1}^{m}\left\|A_{i}^{T}\right\|^{2}\left|\lambda_{i}-\mu_{i}\right|^{2} \leq \sum_{i=1}^{m} d_{i} h_{i}\left\|\bar{u}-\sum_{p=1}^{m} v_{p}\right\|^{2},
\end{aligned}
$$

where $d_{i}$ is the number of arcs incident to node $i$. This shows that (5) holds with $C_{1}=\sqrt{\sum_{i=1}^{m} d_{i} h_{i}}$. Notice that $\sum_{i=1}^{m} d_{i}=2 n$ and $h_{i}$ is at most the diameter of the spanning tree. Since the choice of the spanning tree is arbitrary, we can choose it to minimize $C_{1}$. Also, $A_{i} A_{j}^{T}=0$ if and only if nodes $i$ and $j$ are not joined by an arc, so $\hat{c}=\max \left\{d_{1}, \ldots, d_{m}\right\}$ and the coloring of $K_{1}, \ldots, K_{m}$ is equivalent to graph coloring on the diagraph.

In the above case of a nonlinear network flow problem, if $G$ is also separable in the sense that $G(x)=\sum_{j=1}^{n} G_{j}\left(x_{j}\right)$ for all $x=\left(x_{j}\right)_{j=1}^{n}$ and $G_{j}: \Re \mapsto \Re$, then $\pi_{i}\left(u_{1}, \ldots, u_{m}\right)$ given by (9) depends on only those $u_{k}$ for which node $k$ is a neighbor of node $i$ and the asynchronous method (10)-12) reduces to the asynchronous network relaxation method studied in [5, §7.2.3] and [56. It is known that iterates generated by this method converge for any stepsize $\gamma \in(0,1)$, assuming $G^{*}$ is convex differentiable and (41) has a solution $(G$ need not be defined everywhere on $\Re^{n}$ and $\left(G^{*}\right)^{\prime}$ need not be strongly monotone or Lipschitz continuous). However, no rate of convergence result was known. By applying Theorem 1, we obtain that this method has a linear rate of convergence, assuming $\left(G^{*}\right)^{\prime}$ is strongly monotone and Lipschitz continuous and the stepsize is sufficiently small. 


\section{Applications to partial differential equations WITHOUT CONSTRAINTS}

In this section we consider the Sobolev space $V=H_{0}^{1}(\Omega)=\left\{v \in H^{1}(\Omega)\right.$ : $v=0$ on $\partial \Omega\}$ with duality pairing $\langle u, v\rangle=\int_{\Omega}\left(\sum_{i=1}^{d} \partial_{i} u \partial_{i} v+u v\right) d x$ and norm $\|v\|=\|v\|_{H^{1}(\Omega)}=\langle v, v\rangle^{\frac{1}{2}}$, where $\Omega$ is an open, bounded, and connected subset of $\Re^{d}$ with Lipschitz continuous boundary $\partial \Omega, H^{1}(\Omega)=\left\{v \in L^{2}(\Omega): \partial_{i} v \in\right.$ $\left.L^{2}(\Omega), i=1, \ldots, d\right\}$, and $\partial_{i} v$ is the locally Lebesgue integrable real function defined on $\Omega$ satisfying $\int_{\Omega} \partial_{i} v \phi d x=-\int_{\Omega} v \frac{\partial \phi}{\partial x_{i}} d x$ for all $\phi \in C_{0}^{\infty}(\Omega)=\left\{\phi \in C^{\infty}(\Omega)\right.$ : $\phi$ has compact support\} [17, pp. 10-13]. We will consider two nonlinear elliptic partial differential equations formulated as the minimization problem (2) and, for each, we will consider the space decomposition (4) corresponding to, respectively, DD and MG methods, and we will develop corresponding estimates for $C_{1}$ in (5), for $C_{2}$ in (6) and for $c$ in (17)-(9). Throughout, we denote $|x|=\left(\sum_{i=1}^{d} x_{i}^{2}\right)^{\frac{1}{2}}$ for any $x=\left(x_{i}\right)_{i=1}^{d} \in \Re^{d}$.

The first partial differential equation corresponds to the minimization problem (2) with

$$
K=H_{0}^{1}(\Omega),\left\langle F^{\prime}(u), v\right\rangle=\int_{\Omega}\left(\sum_{i=1}^{d} a_{i}(x, u, \nabla u) \partial_{i} v+a_{0}(x, u, \nabla u) v-f v\right) d x,
$$

where $f \in L^{2}(\Omega)$ and $\nabla u=\left(\partial_{i} u\right)_{i=1}^{d}$ is the gradient of $u$ [18, p. 302]. It is assumed that each nonlinear coefficient $a_{i}(x, p)$ is a real-valued function of $x=\left(x_{j}\right)_{j=1}^{d}$ and $p=\left(p_{k}\right)_{k=0}^{d}$ and is sufficiently smooth in the sense that

$$
\begin{gathered}
a_{i} \in C^{1}\left(\Omega \times \Re^{d+1}\right), \\
\max _{\substack{j=1,2, \ldots d \\
k=0,1, \cdots, d}}\left\{\left|a_{i}(x, p)\right|,\left|\frac{\partial a_{i}}{\partial x_{j}}(x, p)\right|,\left|\frac{\partial a_{i}}{\partial p_{k}}(x, p)\right|\right\} \leq L
\end{gathered}
$$

for all $(x, p) \in \Omega \times \Re^{d+1}$ and $i=0,1, \ldots, d$, with $L$ a constant. In addition, the matrix $\left[\frac{\partial a_{i}}{\partial p_{k}}(x, p)\right]_{i, k=0}^{d}$ is assumed to be uniformly positive definite, i.e.,

$$
\sum_{i=0}^{d} \sum_{k=0}^{d} \frac{\partial a_{i}}{\partial p_{k}}(x, p) \xi_{i} \xi_{k} \geq \sigma \sum_{i=0}^{d} \xi_{i}^{2}, \quad \forall \xi_{i} \in \Re, i=0,1, \ldots, d,
$$

for all $(x, p) \in \Omega \times \Re^{d+1}$, with $\sigma>0$ a constant. Under these assumptions, the problem (2), which has the equation formulation

$$
\left\langle F^{\prime}(u), v\right\rangle=0, \quad \forall v \in H_{0}^{1}(\Omega),
$$

is well posed and has a unique solution $u \in H_{0}^{1}(\Omega)$ (see [18, p. 302] and [32]). Moreover, straightforward calculation shows that

$$
\begin{aligned}
\left\langle F^{\prime}(u)-F^{\prime}(v), u-v\right\rangle & \geq \sigma\|u-v\|^{2}, \\
\left\langle F^{\prime}(u)-F^{\prime}(v), w\right\rangle & \leq L(d+1)\|u-v\|\|w\|,
\end{aligned}
$$

for all $u, v, w \in H^{1}(\Omega)$, so $F^{\prime}$ is strongly monotone and Lipschitz continuous.

The second partial differential equation corresponds to the minimization problem (2) with

$$
K=H_{0}^{1}(\Omega), \quad F(v)=\int_{\Omega}\left(\frac{1}{2}|\nabla v|^{2}+\frac{1}{4} v^{4}-f v\right) d x
$$


where $f \in L^{2}(\Omega)$ and $d \in\{2,3\}$. The corresponding equation is the simplified Ginzburg-Landau equation for superconductivity:

$$
\begin{aligned}
-\Delta u+u^{3} & =f \text { in } \Omega, \\
u & =0 \text { on } \partial \Omega,
\end{aligned}
$$

where $u$ is the wave function which is valid in the absence of internal magnetic field [54], and $\Delta u=\sum_{i=1}^{d} \partial_{i}\left(\partial_{i} u\right)$ denotes the Laplacian of $u$. Notice that $F^{\prime}$ has the form (44), with $a_{0}(x, p)=p_{0}^{3}$ and $a_{i}(x, p)=p_{i}, i=1, \ldots, d$, which does not satisfy (47). Nevertheless, straightforward calculation shows

$$
\begin{aligned}
\left\langle F^{\prime}(u)-F^{\prime}(v), u-v\right\rangle & =\int_{\Omega}|\nabla u-\nabla v|^{2}+\left(u^{3}-v^{3}\right)(u-v) d x \\
& \geq \int_{\Omega}|\nabla u-\nabla v|^{2} d x=|u-v|_{1, \Omega}^{2}
\end{aligned}
$$

for all $u, v \in H^{1}(\Omega)$. Since the semi-norm $|\cdot|_{1, \Omega}$ is equivalent to the norm $\|\cdot\|$ on $H_{0}^{1}(\Omega)$ [17, p. 12], this shows $F^{\prime}$ is strongly monotone on $H_{0}^{1}(\Omega)$.

In subsections 7.1 and 7.2 below, we will study asynchronous DD and MG methods for solving the above two equations (48) and (51). We will analyze the convergence rate of the methods by estimating the constants $C_{1}, C_{2}$ and $c$ for the corresponding space decomposition of the finite element approximation subspace and then applying Theorem 1. In particular, we will show that the above two equations can be solved in parallel with a convergence factor that is independent of the finite element mesh size $h$, i.e., the number of iterations to reach a desired solution accuracy is independent of $h$.

\subsection{Domain decomposition methods.}

7.1.1. Decomposition of the domain $\Omega$. In DD methods, the domain $\Omega$ is decomposed into the disjoint union of subdomains $\Omega_{i}, i=1, \ldots, m$, and their boundary, i.e., $\Omega \cup \partial \Omega=\bigcup_{i=1}^{m}\left(\Omega_{i} \cup \partial \Omega_{i}\right)$ and $\Omega_{i} \cap \Omega_{j}=\emptyset$ for $i \neq j$. This is illustrated in Figure 1 where a rectangular-shaped domain in $\Re^{2}$ is decomposed into the disjoint union of $m=25$ rectangular-shaped subdomains and their boundary. The subdomains, which are assumed to form a regular quasi-uniform division (see p. 124 and Eq. (3.2.28) of [17] for definitions) with a specified maximum diameter of $H$, are the finite elements of the coarse mesh. To form the fine mesh for the finite element approximations, we further divide each $\Omega_{i}$ into finite elements of size (i.e., maximum diameter) $h$ such that all the fine-mesh elements together form a regular finite element division of $\Omega$. We denote this fine division by $\mathcal{T}_{h}$. For each $\Omega_{i}$, we consider an enlarged subdomain $\Omega_{i}^{\delta}=\left\{e \in \mathcal{T}_{h}: \operatorname{dist}\left(e, \Omega_{i}\right) \leq \delta\right\}$, where $\operatorname{dist}\left(e, \Omega_{i}\right)=\min _{x \in e, y \in \Omega_{i}}|x-y|$. The union of $\Omega_{i}^{\delta}, i=1, \ldots, m$, covers $\Omega$ with overlap proportional to $\delta$. Let $K_{0} \subset H_{0}^{1}(\Omega)$ and $K \subset H_{0}^{1}(\Omega)$ denote the continuous, piecewise $r$ th-order polynomial $(r \geq 1)$ finite element subspaces, with zero trace on $\partial \Omega$, over the $H$-level and $h$-level subdivisions of $\Omega$, respectively. For $i=1, \ldots, m$, let $K_{i}$ denote the continuous, piecewise $r$ th-order polynomial finite element subspace with zero trace on the boundary $\partial \Omega_{i}^{\delta}$ and extended to have zero value outside $\Omega_{i}^{\delta} \cup \partial \Omega_{i}^{\delta}$. Then $K_{i}^{\ominus}=K_{i}$ for $i=0,1, \ldots, m$, and it can be shown that

$$
K=\sum_{i=0}^{m} K_{i} .
$$




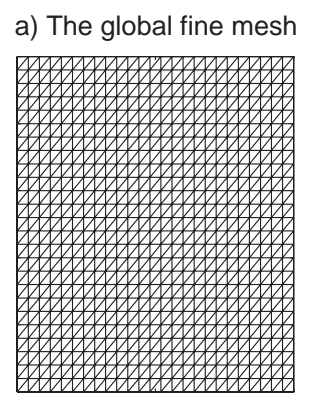

b) Color 0: the coarse mesh

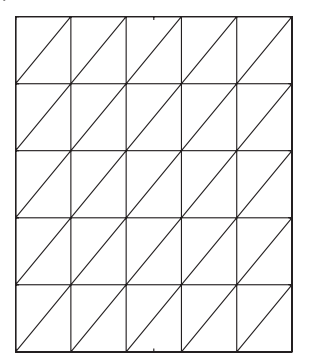

d) Color 2 subdomains

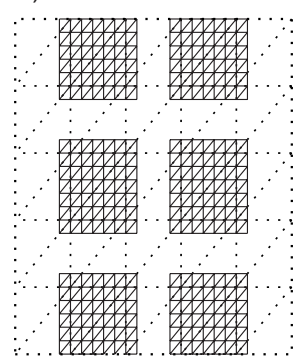

e) Color 3 subdomains

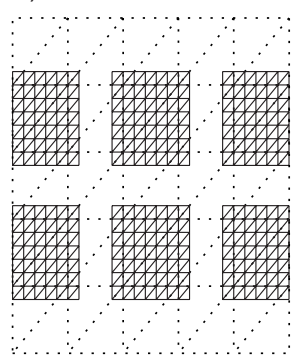

c) Color 1 subdomains

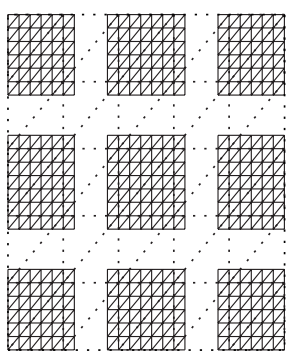

f) Color 4 subdomains

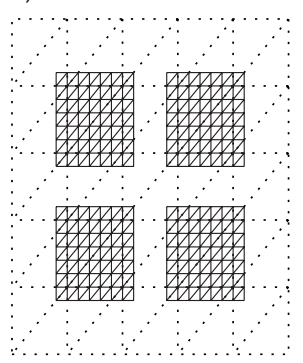

FigURE 1. Decomposition of a rectangular-shaped domain in $\Re^{2}$.

Thus the space decomposition (4), with summation index from 0 to $m$, holds. We assume that the overlapping subdomains are chosen such that each subdomain $\Omega_{i}^{\delta}$ and its corresponding finite element subspace $K_{i}$ can be painted one of $n_{c}$ colors (numbered from 1 to $n_{c}$ ), with subdomains painted the same color being pairwise nonintersecting. The coarse mesh and its corresponding subspace $K_{0}$ are painted the color 0 . Moreover, $n_{c}$ should be independent of $h$. For general domain $\Omega$, finding overlapping subdomains with such property is nontrivial. If $\Omega$ is the Cartesian product of intervals, we can easily find overlapping subdomains with $n_{c}=2$ if $d=1$, and $n_{c} \leq 4$ if $d=2$, and $n_{c} \leq 6$ if $d=3$. For the example of Figure 1 $d=2$ and $n_{c}=4$. Then the total number of colors needed for (7) and (9) to hold is $c=n_{c}+1$.

7.1.2. Estimating $C_{1}$ for equations (48) and (51). Let $\left\{\theta_{i}\right\}_{i=1}^{m}$ be a smooth partition of unity with respect to $\left\{\Omega_{i}\right\}_{i=1}^{m}$, i.e., $\theta_{i} \in C^{\infty}(\Omega)$ with $\theta_{i} \geq 0, \theta_{i}=0$ outside of $\Omega_{i}$, and $\sum_{i=1}^{m} \theta_{i}=1$. Let $I_{h}$ be the finite element interpolation mapping onto $K$ which uses the function values at the $h$-level nodes. For any $v \in K$, let $v_{0}$ be the projection in the $L^{2}$-norm of $v$ onto $K_{0}$, i.e., $v_{0} \in K_{0}$ and $\int_{\Omega}\left(v_{0}-v\right) \phi d x=0$ for all $\phi \in K_{0}$, and let $v_{i}=I_{h}\left(\theta_{i}\left(v-v_{0}\right)\right)$. Then, it can be seen that $v_{i} \in K_{i}$ for $i=0,1, \ldots, m$ and satisfy $v=\sum_{i=0}^{m} v_{i}$ [45, pp. 163-165], [57, p. 607]. Moreover, by further choosing $\theta_{i}$ so that $\left|\nabla \theta_{i}\right|$ has a certain boundedness property, it was recently shown in [53, Lem. 4.1] that, for any $s \geq 1$,

$$
\left(\sum_{i=0}^{m}\left\|v_{i}\right\|^{s}\right)^{\frac{1}{s}} \leq C c^{\frac{1}{s}}\left(1+\left(\frac{H}{\delta}\right)^{\frac{1}{2}}\right)\|v\|,
$$


where $C$ is a constant independent of the mesh parameters and $m$. Taking $s=2$ and using the subspace nature of $K_{i}$, we obtain that, for any $v_{i} \in K_{i}, i=0,1, \ldots, m$, there exists $\bar{u}_{i} \in K_{i}$ satisfying (5) (with summation index from 0 to $m$ ), where

$$
C_{1}=C \sqrt{c}\left(1+\left(\frac{H}{\delta}\right)^{\frac{1}{2}}\right) .
$$

(also see [14. Thm. 16] and a work of Dryja and Widlund cited therein for related results). By choosing the overlapping size $\delta$ proportional to the coarse-mesh size $H$, the constant $C_{1}$ will be independent of the mesh parameters and the number of subdomains $m$.

7.1.3. Estimating $C_{2}$ for equations (48) and (51). Consider $F$ given by (50), associated with the equation (51). By the mean value theorem, for any $u \in \Re, v \in \Re$, we have $\left|u^{3}-v^{3}\right|=3|\theta u+(1-\theta) v|^{2}|u-v| \leq 3(|u|+|v|)^{2}|u-v| \leq 6\left(|u|^{2}+|v|^{2}\right)|u-v|$ for some $\theta \in[0,1]$. Thus, using the continuous embedding of $H^{1}(\Omega)$ in $L^{p}(\Omega)$ for $p<2 d /(d-2)$ and $d=2,3$ (see [17, p. 114], [24, p. 21]), we have for any $u, v \in H^{1}(\Omega)$ and any subdomain $\Omega^{\prime}$ of $\Omega$ that $u, v \in L^{4}(\Omega)$ and

$$
\begin{aligned}
\left|\int_{\Omega^{\prime}}\left(u^{3}-v^{3}\right) w d x\right| & \leq 6 \int_{\Omega^{\prime}}|u|^{2}\left|u-v\left\|\left.w|+| v\right|^{2}|u-v \| w| d x\right.\right. \\
& \leq 6\left(\left(\int_{\Omega^{\prime}}|u|^{4} d x\right)^{\frac{1}{2}}+\left(\int_{\Omega^{\prime}}|v|^{4} d x\right)^{\frac{1}{2}}\right)\left(\int_{\Omega^{\prime}}|u-v|^{2}|w|^{2} d x\right)^{\frac{1}{2}} \\
& \leq 6\left(\|u\|_{L^{4}\left(\Omega^{\prime}\right)}^{2}+\|v\|_{L^{4}\left(\Omega^{\prime}\right)}^{2}\right)\|u-v\|_{L^{4}\left(\Omega^{\prime}\right)}\|w\|_{L^{4}\left(\Omega^{\prime}\right)} \\
& \leq C\left(\|u\|_{H^{1}\left(\Omega^{\prime}\right)}^{2}+\|v\|_{H^{1}\left(\Omega^{\prime}\right)}^{2}\right)\|u-v\|_{H^{1}\left(\Omega^{\prime}\right)}\|w\|_{H^{1}\left(\Omega^{\prime}\right)},
\end{aligned}
$$

where $C$ depends only on the embedding constant. Also, define $\Omega_{0}^{\delta}=\Omega$ for convenience, so that every $v \in K_{i}$ vanishes outside of $\Omega_{i}^{\delta}(i=0,1, \ldots, m)$. Then, for $F$ given by (50), we have from the above inequality that, for $i, j=0,1, \ldots, m$,

$$
\begin{aligned}
a_{i j}= & \left\langle F^{\prime}\left(w_{i j}+u_{i j}\right)-F^{\prime}\left(w_{i j}\right), v_{i}\right\rangle \\
= & \int_{\Omega_{i}^{\delta} \cap \Omega_{j}^{\delta}}\left(\nabla u_{i j}\right)^{T} \nabla v_{i}+u_{i j} v_{i}+\left(\left(w_{i j}+u_{i j}\right)^{3}-w_{i j}^{3}\right) v_{i} d x \\
\leq & \left(1+C\left\|w_{i j}+u_{i j}\right\|_{H^{1}\left(\Omega_{i}^{\delta} \cap \Omega_{j}^{\delta}\right)}^{2}+C\left\|w_{i j}\right\|_{H^{1}\left(\Omega_{i}^{\delta} \cap \Omega_{j}^{\delta}\right)}^{2}\right) \\
& \cdot\left\|u_{i j}\right\|_{H^{1}\left(\Omega_{i}^{\delta} \cap \Omega_{j}^{\delta}\right)}\left\|v_{i}\right\|_{H^{1}\left(\Omega_{i}^{\delta} \cap \Omega_{j}^{\delta}\right)},
\end{aligned}
$$

for any $w_{i j} \in K, u_{i j} \in K_{j}, v_{i} \in K_{i}$, with $a_{i j}=0$ whenever $\Omega_{i}^{\delta} \cap \Omega_{j}^{\delta}=\emptyset$. Assume there exists a constant $\alpha>0$ such that $\left\|w_{i j}+u_{i j}\right\|_{H^{1}\left(\Omega_{i}^{\delta} \cap \Omega_{j}^{\delta}\right)}^{2}+\left\|w_{i j}\right\|_{H^{1}\left(\Omega_{i}^{\delta} \cap \Omega_{j}^{\delta}\right)}^{2} \leq \alpha$ for $i, j=0,1, \ldots, m$. Also, for $i, j=1, \ldots, m$, let $\epsilon_{i j}=0$ if $\Omega_{i}^{\delta} \cap \Omega_{j}^{\delta}=\emptyset$ and otherwise let $\epsilon_{i j}=1$. Let $\hat{c}$ be the smallest integer such that every subdomain intersects at most $\hat{c}$ other subdomains. It is not difficult to show that the symmetric matrix $\mathcal{E}=\left[\epsilon_{i j}\right]_{i, j=1}^{m}$ has the following estimate of its spectral radius (see [53. Corollary 4.1] for a proof):

$$
\rho(\mathcal{E}) \leq \max _{i=1, \ldots, m} \sum_{j=1}^{m} \epsilon_{i j} \leq \hat{c} .
$$


This together with the estimate (52) yields

$$
\begin{aligned}
\sum_{i=1}^{m} \sum_{j=1}^{m} a_{i j} & \leq(1+C \alpha) \sum_{i=1}^{m} \sum_{j=1}^{m} \epsilon_{i j}\left\|u_{i j}\right\|\left\|v_{i}\right\| \\
& \leq(1+C \alpha) \sum_{i=1}^{m} \sum_{j=1}^{m} \epsilon_{i j} \max _{i=1, \ldots, m}\left\|u_{i j}\right\|\left\|v_{i}\right\| \\
& =(1+C \alpha) \hat{c}\left(\sum_{j=1}^{m} \max _{i=1, \ldots, m}\left\|u_{i j}\right\|^{2}\right)^{\frac{1}{2}}\left(\sum_{i=1}^{m}\left\|v_{i}\right\|^{2}\right)^{\frac{1}{2}}
\end{aligned}
$$

Next, by using the fact $\Omega_{j}^{\delta}, j \in I(k)$, are disjoint subsets of $\Omega$ for $k=1, \ldots, c$, the estimate (52) yields

$$
\begin{aligned}
\sum_{j=1}^{m} a_{0 j} & \leq(1+C \alpha) \sum_{j=1}^{m}\left\|u_{0 j}\right\|\left\|v_{0}\right\|_{H^{1}\left(\Omega_{j}^{\delta}\right)} \\
& \leq(1+C \alpha)\left(\sum_{j=1}^{m}\left\|u_{0 j}\right\|^{2}\right)^{\frac{1}{2}}\left(\sum_{j=1}^{m}\left\|v_{0}\right\|_{H^{1}\left(\Omega_{j}^{\delta}\right)}^{2}\right)^{\frac{1}{2}} \\
& \leq(1+C \alpha) \sqrt{c}\left(\sum_{j=1}^{m}\left\|u_{0 j}\right\|^{2}\right)^{\frac{1}{2}}\left\|v_{0}\right\|, \quad \forall u_{0 j} \in K_{j}, \forall v_{0} \in K_{0} .
\end{aligned}
$$

Similar to the above argument, the estimate (52) gives

$$
\begin{aligned}
\sum_{i=1}^{m} a_{i 0} & \leq(1+C \alpha) \sum_{i=1}^{m}\left\|u_{i 0}\right\|_{H^{1}\left(\Omega_{i}^{\delta}\right)}\left\|v_{i}\right\| \\
& \leq(1+C \alpha)\left(\sum_{i=1}^{m}\left\|u_{i 0}\right\|_{H^{1}\left(\Omega_{i}^{\delta}\right)}^{2}\right)^{\frac{1}{2}}\left(\sum_{i=1}^{m}\left\|v_{i}\right\|^{2}\right)^{\frac{1}{2}}, \quad \forall u_{i 0} \in K_{0}, \forall v_{i} \in K_{i} .
\end{aligned}
$$

We combine these estimates to obtain

$$
\begin{aligned}
\sum_{i=0}^{m} \sum_{j=0}^{m} a_{i j}= & a_{00}+\sum_{j=1}^{m} a_{0 j}+\sum_{i=1}^{m} \sum_{j=1}^{m} a_{i j}+\sum_{i=1}^{m} a_{i 0} \\
\leq & (1+C \alpha)\left\|u_{00}\right\|\left\|v_{0}\right\|+(1+C \alpha) \sqrt{c}\left(\sum_{j=1}^{m}\left\|u_{0 j}\right\|^{2}\right)^{\frac{1}{2}}\left\|v_{0}\right\| \\
& +(1+C \alpha) \hat{c}\left(\sum_{j=1}^{m} \max _{i=1, \ldots, m}\left\|u_{i j}\right\|^{2}\right)^{\frac{1}{2}}\left(\sum_{i=1}^{m}\left\|v_{i}\right\|^{2}\right)^{\frac{1}{2}} \\
& +(1+C \alpha)\left(\sum_{i=1}^{m}\left\|u_{i 0}\right\|_{H^{1}\left(\Omega_{i}^{\delta}\right)}^{2}\right)^{\frac{1}{2}}\left(\sum_{i=1}^{m}\left\|v_{i}\right\|^{2}\right)^{\frac{1}{2}} \\
\leq & \tilde{C}_{2}\left(\sum_{j=0}^{m} \max _{i=0,1, \ldots, m}\left\|u_{i j}\right\|^{2}\right)^{\frac{1}{2}}\left(\sum_{i=0}^{m}\left\|v_{i}\right\|^{2}\right)^{\frac{1}{2}} \\
& +(1+C \alpha)\left(\sum_{i=1}^{m}\left\|u_{i 0}\right\|_{H^{1}\left(\Omega_{i}^{\delta}\right)}^{2}\left(\sum_{i=1}^{\frac{1}{2}}\left\|v_{i}\right\|^{2}\right)^{\frac{1}{2}}\right.
\end{aligned}
$$

with $\tilde{C}_{2}$ a constant depending on $C \alpha, c, \hat{c}$ only. Compared with (6) (with $i, j=$ $0,1, \ldots, m)$, we see that (54) has an extra term on the right-hand side. In the 
appendix, we will show that this extra term does not affect the convergence rate result of Section 4. In particular, we will show that Lemmas 1 and 2 hold with $C_{2}=\tilde{C}_{2}+(1+C \alpha) \sqrt{c}$, so that Theorem 1 is still valid.

For $F$ specified by (44) and associated with the equation (48), it can be similarly proved using (49) that (54) holds, possibly with different constants $C$ and $\alpha$.

Upon applying the asynchronous method (10)-(12) with the above choice of space decomposition and under the assumptions (13)-(14), we obtain a parallel DD method for (48) and (51) whose convergence factor, according to Theorem 1 and the above estimates of $C_{1}$ and $C_{2}$ and assuming the overlapping size $\delta$ is proportional to the coarse mesh size $H$, is independent of the mesh parameters and the number of the subdomains.

\subsection{Multigrid methods.}

7.2.1. Construction of the multigrid subspaces. In MG methods, $\Omega$ is divided into a finite element triangulation $\mathcal{T}$ by a successive refinement process. More precisely, we have $\mathcal{T}=\mathcal{T}_{J}$ for some $J>1$, where $\mathcal{T}_{k}, k=1, \ldots, J$, is a nested sequence of regular quasi-uniform triangulation, i.e., $\mathcal{T}_{k}$ is a collection of simplexes $\mathcal{T}_{k}=\left\{\tau_{i}^{k}\right\}$ of size (i.e., maximum diameter) $h_{k}$ such that $\Omega=\bigcup_{i} \tau_{i}^{k}$ and for which the quasiuniformity constants are independent of $k$ [17, Eq. (3.2.28)] and with each simplex in $\mathcal{T}_{k-1}$ being the union of simplexes in $\mathcal{T}_{k}$. We further assume that there is a constant $r<1$, independent of $k$, such that $h_{k}$ is proportional to $r^{2 k}$.

For example, in the two-dimensional case of $d=2$, if we construct $\mathcal{T}_{k}$ by connecting the midpoints of the edges of the triangles of $\mathcal{T}_{k-1}$, with $\mathcal{T}_{1}$ being the given coarsest initial triangulation, the resulting sequence of triangulation is quasiuniform and $r=1 / \sqrt{2}$ (see Figure 2). Corresponding to each triangulation $\mathcal{T}_{k}$, we define the finite element subspace:

$$
\mathcal{M}_{k}=\left\{v \in H_{0}^{1}(\Omega):\left.\quad v\right|_{\tau} \in \mathcal{P}_{1}(\tau), \quad \forall \tau \in \mathcal{T}_{k}\right\},
$$
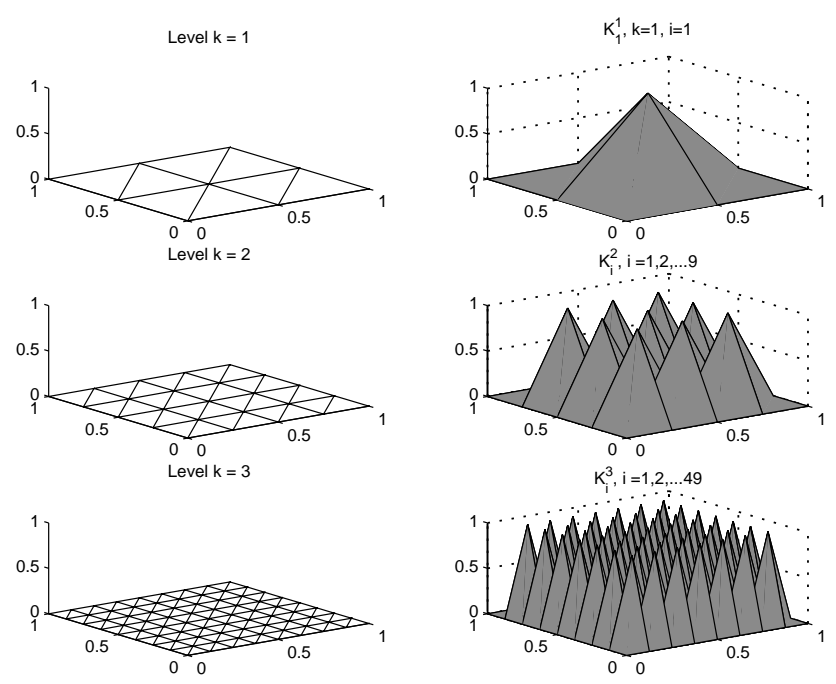

FIGURE 2. The multigrid mesh and basis functions. 
where $\mathcal{P}_{1}(\tau)$ denotes the space of real-valued linear functions of $d$ real variables defined on $\tau$. We associate with $\mathcal{M}_{k}$ a nodal basis, denoted by $\left\{\phi_{i}^{k}\right\}_{i=1}^{n_{k}}$, that satisfies $\phi_{i}^{k} \in \mathcal{M}_{k}$ and

$$
\phi_{i}^{k}\left(x_{j}^{k}\right)=\delta_{i j}, \quad \text { the Kronecker function, }
$$

where $\left\{x_{i}^{k}\right\}_{i=1}^{n_{k}}$ is the set of all interior nodes of the triangulation $\mathcal{T}_{k}$. For each such nodal basis function, we define the one-dimensional subspace

$$
K_{i}^{k}=\operatorname{span}\left(\phi_{i}^{k}\right) .
$$

Then, $\left(K_{i}^{k}\right)^{\ominus}=K_{i}^{k}$ and we have the space decomposition

$$
K=\sum_{k=1}^{J} \sum_{i=1}^{n_{k}} K_{i}^{k} \quad \text { with } \quad K=\mathcal{M}_{J} .
$$

On each level $k$, we color the nodes of $\mathcal{T}_{k}$ so that neighboring nodes are always of a different color. The number of colors needed for a regular mesh is a constant independent of the mesh parameters, which we denote by $n_{c}$. Then the total number of colors needed for (7) and (9) (with summation indices adjusted accordingly) to hold is $c=n_{c} J$.

7.2.2. Estimating $C_{1}$ for equations (48) and (51). Let $Q_{k}$ be the projection in the $L^{2}$-norm onto the subspace $\mathcal{M}_{k}$, which is well defined on $H_{0}^{1}(\Omega) \subset L^{2}(\Omega)$. For any $v \in K$, let $v^{k}=\left(Q_{k}-Q_{k-1}\right) v, k=1, \ldots, J$. Then, by Prop. 8.6 in [57, p. 611], we have

$$
\sum_{k=1}^{J}\left\|v^{k}\right\|^{2} \leq C_{0}\|v\|^{2},
$$

where $C_{0}$ is a constant independent of the mesh parameters and $J$. By further decomposing each $v^{k}$ as

$$
v^{k}=\sum_{i=1}^{n_{k}} v_{i}^{k} \quad \text { with } \quad v_{i}^{k}=v^{k}\left(x_{i}^{k}\right) \phi_{i}^{k},
$$

the above estimate can be refined to show that

$$
v=\sum_{k=1}^{J} \sum_{i=1}^{n_{k}} v_{i}^{k} \quad \text { and } \quad \sum_{k=1}^{J} \sum_{i=1}^{n_{k}}\left\|v_{i}^{k}\right\|^{2} \leq C\|v\|^{2},
$$

where $C$ is a constant independent of the mesh parameters and the number of levels $J$ [53, §4.2]. Thus, for any $v_{i}^{k} \in K_{i}^{k}, i=1, \ldots, n_{k}, k=1, \ldots, J$, there exists $\bar{u}_{i}^{k} \in K_{i}^{k}$ satisfying (5) (with summation indices adjusted accordingly), where $C_{1}=\sqrt{C}$.

7.2.3. Estimating $C_{2}$ for equations (48) and (51). Let $\Lambda_{i}^{k}$ denote the support set of the basis function $\phi_{i}^{k}$, for all $i$ and $k$. Also, recall the constant $r<1$ defined earlier. Then, for any $k<l$ and $1 \leq i \leq n_{k}, 1 \leq j \leq n_{l}$, the following estimate

$$
\|u\|_{H^{1}\left(\Lambda_{i}^{k} \cap \Lambda_{j}^{l}\right)} \leq C_{0} r^{d(l-k)}\|u\|, \quad \forall u \in K_{i}^{k},
$$


can be shown, where $C_{0}$ is a constant independent of the mesh parameters and $J$ [53, Eq. (49)]. Then, for $F$ given by (50), we obtain as in (52) that

$$
\begin{aligned}
& \left\langle F^{\prime}(w+u)-F^{\prime}(w), v\right\rangle \\
& \leq\left(1+C\|w+u\|_{H^{1}\left(\Lambda_{i}^{k} \cap \Lambda_{j}^{l}\right)}^{2}+C\|w\|_{H^{1}\left(\Lambda_{i}^{k} \cap \Lambda_{j}^{l}\right)}^{2}\right)\|u\|_{H^{1}\left(\Lambda_{i}^{k} \cap \Lambda_{j}^{l}\right)}\|v\|_{H^{1}\left(\Lambda_{i}^{k} \cap \Lambda_{j}^{l}\right)} \\
& \leq\left(1+C\|w+u\|_{H^{1}\left(\Lambda_{i}^{k} \cap \Lambda_{j}^{l}\right)}+C\|w\|_{H^{1}\left(\Lambda_{i}^{k} \cap \Lambda_{j}^{l}\right)}\right) C_{0} r^{d(l-k)}\|u\|\|v\|, \\
& \quad \forall w \in K, u \in K_{i}^{k}, v \in K_{j}^{l},
\end{aligned}
$$

where $C$ is the embedding constant. For any $i, j, k, l$, defining

$$
\varepsilon_{i, j}^{k, l}= \begin{cases}C_{0} \gamma^{d|l-k|}, & \text { if } \operatorname{supp}\left(\phi_{i}^{k}\right) \cap \operatorname{supp}\left(\phi_{j}^{l}\right) \neq \emptyset \\ 0, & \text { otherwise. }\end{cases}
$$

Assuming there exists a constant $\alpha>0$ such that $\left\|w_{i, j}^{k, l}+u_{i, j}^{k, l}\right\|^{2}+\left\|w_{i, j}^{k, l}\right\|^{2} \leq \alpha$ for all $i, j, k, l$, the estimate (55) then yields

$$
\begin{aligned}
& \sum_{k=1}^{J} \sum_{i=1}^{n_{k}} \sum_{l=1}^{J} \sum_{j=1}^{n_{l}}\left\langle F^{\prime}\left(w_{i, j}^{k, l}+u_{i, j}^{k, l}\right)-F^{\prime}\left(w_{i, j}^{k, l}\right), v_{i}^{k}\right\rangle \\
& \quad \leq C_{0}(1+C \alpha) \sum_{i, k} \sum_{j, l} \varepsilon_{i, j}^{k, l}\left\|u_{i, j}^{k, l}\right\|\left\|v_{i}^{k}\right\| \\
& \quad \leq C_{0}(1+C \alpha) \sum_{k=1}^{J} \sum_{i=1}^{n_{k}} \sum_{l=1}^{J} \sum_{j=1}^{n_{l}} \varepsilon_{i, j}^{k, l} \max _{i, k}\left\|u_{i, j}^{k, l}\right\| \cdot\left\|v_{i}^{k}\right\|, \\
& \forall u_{i, j}^{k, l} \in K_{j}^{l}, \forall v_{i}^{k} \in K_{i}^{k} .
\end{aligned}
$$

With proper ordering of the indices, the matrix $\mathcal{E}=\left[\varepsilon_{i, j}^{k, l}\right]$ is symmetric and its spectral radius $\rho(\mathcal{E})$ has been shown to be less than a constant independent of the mesh parameters and the number of levels [45, pp. 182-184]. Therefore,

$$
\begin{aligned}
& \sum_{k=1}^{J} \sum_{i=1}^{n_{k}} \sum_{l=1}^{J} \sum_{j=1}^{n_{l}}\left\langle F^{\prime}\left(w_{i, j}^{k, l}+u_{i, j}^{k, l}\right)-F^{\prime}\left(w_{i, j}^{k, l}\right), v_{i}^{k}\right\rangle \\
& \quad \leq C_{0}(1+C \alpha) \rho(\mathcal{E})\left(\sum_{l=1}^{J} \sum_{j=1}^{n_{l}} \max _{i, k}\left\|u_{i, j}^{k, l}\right\|^{2}\right)^{\frac{1}{2}}\left(\sum_{k=1}^{J} \sum_{i=1}^{n_{k}}\left\|v_{i}^{k}\right\|^{2}\right)^{\frac{1}{2}},
\end{aligned}
$$

which shows that (6) holds, with the constant $C_{2}=C_{0}(1+C \alpha) \rho(\mathcal{E})$ independent of the mesh parameters and the number of levels for the MG approximation.

For $F$ specified by (44), it can be similarly proved that (6) holds with $C_{2}$ some constant independent of the mesh parameters and the number of levels.

Upon applying the asynchronous method (10)-(12) with the above choice of space decomposition and under the assumptions (13)-(14), we obtain a parallel MG method for (48) and (51) whose convergence factor, according to the above estimates of $C_{1}$ and $C_{2}$ and Theorem 1, is independent of the mesh parameters. This method generalizes the BPX multigrid method proposed in [10], which was used as a preconditioner for linear elliptic problems. Here, the parallel MG method is used as a solver and is applicable not only to linear, but also to nonlinear elliptic problems. And it further allows for asynchronous updates. 


\section{ApPliCATIONS TO OBSTACLE PROBLEMS}

In this section, we will apply our asynchronous algorithm to the obstacle problem

$$
-\Delta u \geq f \text { in } \Omega, \quad u \geq \psi \text { in } \Omega, \quad u=0 \text { on } \partial \Omega,
$$

where $f \in L^{2}(\Omega)$ and $\psi \in H^{2}(\Omega)$ satisfies $\psi \leq 0$ on $\partial \Omega$. This problem is equivalent to (2) with

$$
K=\left\{v \in H_{0}^{1}(\Omega): \quad v \geq \psi \text { a.e. in } \Omega\right\}, \quad F(v)=\int_{\Omega}\left(\frac{1}{2}|\nabla v|^{2}-f v\right) d x .
$$

We will use the overlapping domain decomposition without the coarse mesh. If a coarse mesh is added, it is not known how the coarse mesh obstacle can be chosen to obtain an algorithm whose convergence factor is independent of mesh parameters.

Let $\Omega_{i}^{\delta}$ be defined as in subsection $7.1 i=1, \ldots, m$. Let $\theta_{i}$ be the partition of unity with respect to $\Omega_{i}^{\delta}$ as described in subsection 7.1.2 Accordingly, let $\psi_{i}=$ $I_{h}\left(\theta_{i} \psi\right)$ for all $i$ and let $\psi=\sum_{i=1}^{m} \psi_{i}$, where $I_{h}$ is the interpolation operator using the $h$-level nodal values. Thus, the obstacle function $\psi$ is replaced by its finiteelement interpolation. Defining

$$
K_{i}=\left\{v \in H_{0}^{1}\left(\Omega_{i}^{\delta}\right): \quad v \geq \psi_{i},\left.v\right|_{e} \in P_{1}(e), \forall e \in \mathcal{T}_{h}\right\}
$$

and also assuming that $K$ has been replaced by its finite-element analog, it is easy to see that (4) holds. Suppose we apply the asynchronous algorithm with the initial values $u_{i}(0) \in K_{i}$ chosen to satisfy $\sum_{i=1}^{m} u_{i}(0) \leq \bar{u}$. Then, it can be shown (see, e.g., [1] and [51, §4]) that

$$
\sum_{i=1}^{m} w_{i}\left(t^{i}\right) \leq \bar{u}, \quad \forall t \geq 1
$$

where $w_{i}(\cdot)$ is defined by (12) and $t^{i}$ denotes the greatest element of $T^{i}$ less than $t+B$. Thus (27) remains valid if we assume there exist $\bar{u}_{i} \in K_{i}$ satisfying (5) only for those $v_{i} \in K_{i}$ satisfying $\sum_{i=1}^{m} v_{i} \leq \bar{u}$. Since (27) is the only point in the proofs of Lemmas 1 and 2 where (5) is used, these lemmas and Theorem 1 would remain valid. Under the condition that $\sum_{i=1}^{m} v_{i} \leq \bar{u}$, the constant $C_{1}$ in (5) can be estimated by choosing the partition of unity $\theta_{i}$ to satisfy $\left|\nabla \theta_{i}\right| \leq C / \delta$ and setting

$$
\bar{u}_{i}=v_{i}+\theta_{i}\left(\bar{u}-\sum_{j=1}^{m} v_{j}\right) .
$$

Then, it is straightforward to show that $\bar{u}_{i} \in K_{i}$ and

$$
\sum_{i=1}^{m} \bar{u}_{i}=\bar{u}, \quad \sum_{i=1}^{m}\left\|\bar{u}_{i}-v_{i}\right\|^{2} \leq C\left(1+\delta^{-2}\right)\left\|\bar{u}-\sum_{j=1}^{m} v_{j}\right\|^{2},
$$

with the constant $C$ being independent of $u, v_{i}$, the mesh size $h$, the overlapping size $\delta$, and the number of subdomains $m$ (cf. subsection 7.1.2). The above estimate shows that (5) holds with $C_{1}=\sqrt{C} \sqrt{1+1 / \delta^{2}}$. Also, by dropping the coarse mesh and taking into account the difference between the above $F$ and the $F$ given by (50), we see from the proof of (53) that (6) holds with $C_{2}=(1+C) \hat{c}$, with $C$ an embedding constant. Assuming that $B$ is bounded by a given constant and $\gamma$ is 
bounded by a constant less than 1, we then obtain from the definitions in Lemmas 1 and 2 that

$$
\frac{1}{D} \leq A_{1} \leq D, \quad A_{2} \leq D, \quad A_{3} \leq D\left(1+\delta^{-2}\right), \quad \frac{A_{4}}{A_{3}} \leq D,
$$

for some $D>0$ independent of $h, \delta$ and $m$. Then, the convergence factor given by (38) can be estimated by

$$
\begin{aligned}
\varrho & =\max \left\{1-\frac{\gamma}{1+A_{1}^{-1} A_{3}}+\gamma^{3 / 2} \frac{\left(A_{2}+A_{1} A_{4} / A_{3}\right)}{1+A_{1} A_{3}^{-1}}, \gamma^{1 / 2}\left(A_{1}^{-1}+\gamma^{3 / 2} A_{2} A_{1}^{-1}\right)\right\} \\
& \leq \max \left\{1-\frac{\gamma}{1+D^{2}\left(1+\delta^{-2}\right)}+\gamma^{3 / 2}\left(D+D^{2}\right), \gamma^{1 / 2}\left(D+D^{2}\right)\right\} \\
& \leq \max \left\{1-\frac{\gamma}{D_{1}\left(1+\delta^{-2}\right)}+\gamma^{3 / 2} D_{2}, \gamma^{1 / 2} D_{2}\right\},
\end{aligned}
$$

where we let $D_{1}=1+D^{2}$ and $D_{2}=D+D^{2}$. Thus, for

$$
\gamma<\min \left\{\left(D_{1} D_{2}\left(1+\delta^{-2}\right)\right)^{-2}, D_{2}^{-2}\right\}
$$

we have $\varrho<1$, independent of $h$ and $m$. Finally, we note that the convergence factor for some synchronous overlapping domain decomposition without the coarse mesh has been studied in [1, 60. The schemes obtained from our algorithms are different from those of [1, 60] in the treatment of the subproblem obstacles. The algorithms of [1, 60 use the global obstacle for the subdomain problems. In our algorithms, the subdomain obstacles can be updated dynamically during the iterations.

\section{Appendix}

In this appendix, we show that (54) can be used in place of (6) to prove Lemmas 1 and 2 for the DD method of subsection 7.1 Here, the indices $i$ and $j$ are understood to always range over $0,1, \ldots, m$, instead of $1, \ldots, m$.

First, we note that condition (6) is used only to show (22), (30) and (34) in the proofs. For (22), if we use condition (54) instead of (6), then (22) would have $\tilde{C}_{2}$ in place of $C_{2}$ and would have the following extra term on its right-hand side:

$$
E=(1+C \alpha) \gamma\left(\sum_{i=1}^{m}\left\|u_{0}\left(\tau_{0}^{i}(t)\right)-u_{0}(t)\right\|_{H^{1}\left(\Omega_{i}^{\delta}\right)}^{2}\right)^{\frac{1}{2}}\left(\sum_{i=1}^{m}\left\|s_{i}(t)\right\|^{2}\right)^{\frac{1}{2}} .
$$

Correspondingly, (24) would have $\tilde{C}_{2}$ in place of $C_{2}$ and would have the above extra term on its right-hand side. Using (23) and the fact that $\Omega_{i}^{\delta}, i \in I(k)$, are disjoint subsets of $\Omega$ for $k=1, \ldots, c$, we see that

$$
\begin{aligned}
E & \leq(1+C \alpha) \gamma^{2} \sqrt{B}\left(\sum_{i=1}^{m} \sum_{\tau=t-B+1}^{t-1}\left\|s_{0}(\tau)\right\|_{H^{1}\left(\Omega_{i}^{\delta}\right)}^{2}\right)^{\frac{1}{2}}\left(\sum_{i=1}^{m}\left\|s_{i}(t)\right\|^{2}\right)^{\frac{1}{2}} \\
& \leq(1+C \alpha) \gamma^{2} \sqrt{B c}\left(\sum_{\tau=t-B+1}^{t-1}\left\|s_{0}(\tau)\right\|^{2}\right)^{\frac{1}{2}}\left(\sum_{i=1}^{m}\left\|s_{i}(t)\right\|^{2}\right)^{\frac{1}{2}}
\end{aligned}
$$

which implies that (24) holds with $C_{2}=\tilde{C}_{2}+(1+C \alpha) \sqrt{c}$. The remainder of the proof of Lemma 1 then proceeds as before.

For (30) and (34), a similar argument can be applied to show that Lemma 2 holds with the above choice of $C_{2}$. 


\section{REFERENCES}

1. L. Badea and J. Wang, An additive Schwarz method for variational inequalities, Math. Comp. 69 (2000), 1341-1354. MR 2001a:65075

2. R. Bank, Analysis of a multilevel iterative method for nonlinear finite element equations, Math. Comp. 39 (1982), 453-465. MR 83j:65105

3. R. Bank and T. F. Chan, PLTMGC: a multigrid continuation program for parametrized nonlinear systems, SIAM T. Sci. Statist. Comput., 7 (1986), 540-559. MR 87d:65125

4. D. P. Bertsekas, D. Castañon, J. Eckstein, and S. A. Zenios, Parallel computing in network optimization, Handbooks in Operations Research and Management Science 7: Network Models (Amsterdam) (M. O. Ball, T. L. Magnanti, C. L. Monma, and G. L. Nemhauser, eds.), Elsevier Scientific, 1995, pp. 331-399. CMP 97:04

5. D. P. Bertsekas and J. N. Tsitsiklis, Parallel and distributed computation: Numerical methods, Prentice-Hall, Englewood Cliffs, 1989.

6. $\quad$ Some aspects of parallel and distributed iterative algorithms - a survey, Automatica 27 (1991), 3-21. MR 91m:65137

7. J. Bramble and X. Zhang, The analysis of multigrid methods, Handbook of numerical analysis, Vol. VII, 173-415, North-Holland, Amsterdam, 2000. CMP 2001:08

8. J. H. Bramble, J. E. Pasciak, and A. H. Schatz, The construction of preconditioners for elliptic problems by substructuring. IV, Math. Comp. 53 (1989), 1-24. MR 89m:65098

9. J. H. Bramble, J. E. Pasciak, J. Wang, and J. Xu, Convergence estimates for product iterative methods with applications to domain decomposition, Math. Comp. 57 (1991), 1-21. MR 92d:65094

10. J. H. Bramble, J. E. Pasciak, and J. Xu, Parallel multilevel preconditioners, Math. Comp. 55 (1990), 1-22. MR 90k:65170

11. A. Brandt, Multilevel adaptive solutions to boundary value problems, Math. Comp. 31 (1977), 333-390. MR 55:4714

12. A. Brandt and C. W. Cryer, Multigrid algorithms for the solution of linear complementary problems arising from free boundary problems, SIAM J. Sci. Comput. 4 (1983), 655-684. MR 85h:65261

13. J. V. Burke and P. Tseng, A unified analysis of Hoffman's bound via Fenchel duality, SIAM J. Optim. 6 (1996), 265-282. MR 97c:90120

14. T. F. Chan and T. P. Mathew, Domain decomposition algorithms, Acta Numerica (1994), 61-143. MR 95f:65214

15. T. F. Chan and I. Sharapov, Subspace correction multilevel methods for elliptic eigenvalue problems, Numer. Linear Algebra Appl., 1 (1993), 1-7.

16. D. Chazan and W. L. Miranker, Chaotic relaxation, Lin. Algeb. Appl. 2 (1969), 199-222. MR 40:5114

17. P. G. Ciarlet, The finite element method for elliptic problems, North-Holland, Amsterdam, 1978. MR 58:25001

18. M. Dryja and W. Hackbusch, On the nonlinear domain decomposition method, BIT 37 (1997), 296-311. MR 97m:65100

19. M. Dryja and O. B. Widlund, Towards a unified theory of domain decomposition algorithms for elliptic problems, Third international symposium on domain decomposition methods for partial differential equations (Philadelphia, PA) (T. Chan, R. Glowinski, J. Periaux, and O. B. Widlund, eds.), SIAM, 1990. MR 91m:65294

20. I. Ekeland and R. Temam, Convex analysis and variational problems, North-Holland, Amsterdam, 1976. MR 57:3931b

21. B. H. Elton, An asynchronous domain decomposition method for the two-dimensional Poisson equation, Parallel processing for scientific computing, Vol. I, II (Norfolk, VA, 1993), SIAM, Philadelphia, PA, 1993, pp. 691-695. CMP 93:10

22. A. Frommer, H. Schwandt, and D. B. Szyld, Asychronous weighted additive Schwarz methods, Electronic Trans. Numer. Anal. 5 (1997), 48-61. MR 98c:65046

23. E. Gelman and J. Mandel, On multilevel iterative methods for optimization problems, Math. Programming 48 (1990), 1-17. MR 91b:90180

24. R. Glowinski and P. Le Tallec, Augmented Lagrangian and operator-splitting methods in nonlinear mechanics, SIAM, Philadelphia, 1989. MR 91f:73038

25. W. Hackbusch and H. D. Mittelmann, On multigrid methods for variational inequalities, Numer. Math. 42 (1983), 65-76. MR 84k:49010 
26. W. Hackbusch and A. Reusken, Analysis of a damped nonlinear multilevel method, Numer. Math. 55 (1989), 225-246. MR 90f:65194

27. L. Hart and S. McCormick, Asynchronous multilevel adaptive methods for solving partial differential equations on multiprocessors: basic ideas, Parallel Comput. 12 (1989), no. 2, 131-144. MR 90m:65250

28. R. H. Hoppe, Multigrid solutions to the elastic plastic torsion problem in multiply connected domains, Int. J. Numer. Methods Engin. 26 (1988), 631-646. MR 89a:73001

29. R. H. W. Hoppe, Two-sided approximations for unilateral variational inequalities by multigrid methods, Optimization 16 (1987), 867-881. MR 88m:49004

30. R. H. W. Hoppe and R. Kornhuber, Adaptive multilevel methods for obstacle problems, SIAM J. Numer. Anal. 31 (1994), 301-323. MR 95c:65181

31. R. Kornhuber, Monotone multigrid iterations for elliptic variational inequalities, II, Numer. Math. 72 (1996), 481-499. MR 96k:65081

32. O. A. Ladyzhenskaya and N. N. Uraltseva, Linear and quasilinear elliptic equations, Academic Press, 1968. MR 39:5941

33. P. Le Tallec, Domain decomposition methods in computational mechanics, Comput. Mechan. Adv. 1 (1994), 121-220. MR 95b:65147

34. Z.-Q. Luo and P. Tseng, On the linear convergence of descent methods for convex essentially smooth minimization, SIAM J. Control Optim. 30 (1992), 408-425. MR 93a:90064

35. _ Error bounds and convergence analysis of feasible descent methods: A general approach, Ann. Oper. Res. 46 (1993), 157-178. MR 95a:90138

36. J. Mandel, A multilevel iterative method for symmetric, positive definite linear complementary problems, Appl. Math. Optim. 11 (1984), 77-95. MR 85b:90082

37. S. McCormick and D. Quinlan, Asynchronous multilevel adaptive methods for solving partial differential equations on multiprocessors: performance results, Parallel Comput. 12 (1989), no. 2, 145-156. MR 90m:65251

38. S. F. McCormick, Multilevel adaptive methods for partial differential equations, Frontiers in Applied Mathematics, vol. 6, SIAM, Philadelphia, 1989. MR 91h:65200

39. J.-C. Miellou, L. Giraud, A. Laouar, and P. Spiteri, Subdomain decomposition methods with overlapping and asynchronous iterations, Progress in partial differential equations: the Metz surveys, Longman Sci. Tech., Harlow, 1991, pp. 166-183. MR 94h:65125

40. A. Quarteroni and A. Valli, Domain decomposition methods for partial differential equations, Oxford Science Publications, Oxford, 1999.

41. R. Rannacher, On the convergence of the Newton-Raphson method for strongly nonlinear finite element equations, Nonlinear computational mechanics (P. Wriggers and W. Wagner, eds.), Springer-Verlag, 1991.

42. R. T. Rockafellar, Convex analysis, Princeton University Press, Princeton, 1970. MR 43:445

43. - Network flows and monotropic optimization, Wiley-Interscience, New York, 1984. MR 89d:90156

44. I. Sharapov, Multilevel subspace correction for large scale optimization problems, East-West J. Numer. Math., 9 (2000), 233-252.

45. B. F. Smith, P. E. Bjørstad, and W. D. Gropp, Domain decomposition: Parallel multilevel algorithms for elliptic partial differential equations, Cambridge University Press, Cambridge, 1996. MR 98g:65003

46. A. K. Stagg and G. F. Carey, Asynchronous nonlinear iteration and domain decomposition, Parallel processing for scientific computing (Houston, TX, 1991), SIAM, Philadelphia, PA, 1992, pp. 281-286. CMP 93:05

47. X.-C. Tai, Parallel function decomposition and space decomposition methods with applications to optimisation, splitting and domain decomposition, Preprint No. 231-1992, Institut für Mathematik, TechnischeUniversität Graz (1992), See also http://www.mi.uib.no/ttai.

48. _ Parallel function and space decomposition methods, Finite element methods, fifty years of the Courant element, Lecture notes in pure and applied mathematics (P. Neittaanmäki, ed.), vol. 164, Marcel Dekker inc., 1994, pp. 421-432. See also http://www.mi.uib.no/tai. MR 95d:65008

49. _ Domain decomposition for linear and nonlinear elliptic problems via function or space decomposition, Domain decomposition methods in scientific and engineering computing (Proc. of the 7th international conference on domain decomposition, Penn. State University, 
1993) (D. Keyes and J. Xu, eds.), Contemp. Math. 180, American Mathematical Society, 1994, pp. 355-360. MR 95k:65121

50. — Parallel function decomposition and space decomposition methods: Part II. Space decomposition, Beijing Mathematics 1, part 2 (1995), 135-152, See also http://www.mi.uib.no/rai.

51. Convergence rate analysis of domain decomposition methods for obstacle problems, East-West J. Numer. Math., 9 (2000), 233-253.

52. X.-C. Tai and M. Espedal, Rate of convergence of some space decomposition method for linear and nonlinear elliptic problems, SIAM J. Numer. Anal. 35 (1998), 1558-1570. MR 99k:65101

53. X-C. Tai and J.-C. Xu, Global and uniform convergence of subspace correction methods for some convex optimization problems, Math. Comp., posted on May 11, 2001, PII S00255718(01)01311-4 (to appear in print).

54. M. Tinkham, Introduction to superconductivity, McGraw Hill, New York, 1975.

55. P. Tseng, On the rate of convergence of a partially asynchronous gradient projection algorithm, SIAM J. Optim. 1 (1991), 603-619. MR 92k:90096

56. P. Tseng, D. P. Bertsekas, and J. N. Tsitsiklis, Partially asynchronous, parallel algorithms for network flow and other problems, SIAM J. Control Optim. 28 (1990), 678-710. MR 91e:65075

57. J.-C. Xu, Iterative methods by space decomposition and subspace correction, SIAM Rev. 34 (1992), 581-613. MR 93k:65029

58. (1994), 231-237. MR 94m:65178

59. _ Two-grid discretization techniques for linear and nonlinear PDEs, SIAM J. Numer. Anal. 27 (1996), 1759-1777. MR 97i:65169

60. J. Zeng and S. Zhou, On monotone and geometric convergence of Schwarz methods for twosided obstacle problems, SIAM J. Numer. Anal. 35 (1998), 600-616. MR 99d:65199

Department of Mathematics, University of Bergen, Johannes Brunsgate 12, 5007, Bergen, Norway

E-mail address: Xue-Cheng.Tai@mi.uib.no

Department of Mathematics, Box 354350, University of Washington, Seattle, WashINGTON 98195

E-mail address: tseng@math.washington.edu 